\title{
Increased de novo ceramide synthesis and accumulation in failing myocardium
}

\author{
Ruiping Ji, ${ }^{1}$ Hirokazu Akashi, ${ }^{1}$ Konstantinos Drosatos, ${ }^{2}$ Xianghai Liao, ${ }^{1}$ Hongfeng Jiang, ${ }^{4}$ \\ Peter J. Kennel, ${ }^{1}$ Danielle L. Brunjes, ${ }^{1}$ Estibaliz Castillero, ${ }^{3}$ Xiaokan Zhang, ${ }^{1}$ Lily Y. Deng, ${ }^{1}$ \\ Shunichi Homma, ${ }^{1}$ Isaac J. George, ${ }^{3}$ Hiroo Takayama, ${ }^{3}$ Yoshifumi Naka, ${ }^{3}$ Ira J. Goldberg, ${ }^{4,5}$ \\ and P. Christian Schulze ${ }^{1,6}$ \\ 'Division of Cardiology, Columbia University Medical Center, New York, New York, USA. ${ }^{2}$ Metabolic Biology Laboratory, \\ Temple University School of Medicine, Center for Translational Medicine, Department of Pharmacology, Philadelphia, \\ Pennsylvania, USA. ${ }^{3}$ Division of Cardiothoracic Surgery, Department of Surgery, ${ }^{4}$ Division of Preventive Medicine \\ and Nutrition, Department of Medicine, Columbia University Medical Center, New York, New York, USA. ${ }^{5}$ Division of \\ Endocrinology, Diabetes and Metabolism, New York University Langone Medical Center, New York, New York, USA. \\ ${ }^{6}$ Department of Internal Medicine I, Division of Cardiology, Angiology, Pneumology and Intensive Medical Care, University \\ Hospital Jena, Friedrich-Schiller-University Jena, Jena, Cermany.
}

\begin{abstract}
Abnormal lipid metabolism may contribute to myocardial injury and remodeling. To determine whether accumulation of very long-chain ceramides occurs in human failing myocardium, we analyzed myocardial tissue and serum from patients with severe heart failure (HF) undergoing placement of left ventricular assist devices and controls. Lipidomic analysis revealed increased total and very long-chain ceramides in myocardium and serum of patients with advanced HF. After unloading, these changes showed partial reversibility. Following myocardial infarction (MI), serine palmitoyl transferase (SPT), the rate-limiting enzyme of the de novo pathway of ceramide synthesis, and ceramides were found increased. Blockade of SPT by the specific inhibitor myriocin reduced ceramide accumulation in ischemic cardiomyopathy and decreased C16, C24:1, and C24 ceramides. SPT inhibition also reduced ventricular remodeling, fibrosis, and macrophage content following MI. Further, genetic deletion of the SPTLC2 gene preserved cardiac function following MI. Finally, in vitro studies revealed that changes in ceramide synthesis are linked to hypoxia and inflammation. In conclusion, cardiac ceramides accumulate in the failing myocardium, and increased levels are detectable in circulation. Inhibition of de novo ceramide synthesis reduces cardiac remodeling. Thus, increased de novo ceramide synthesis contributes to progressive pathologic cardiac remodeling and dysfunction.
\end{abstract}

Conflict of interest: The authors have declared that no conflict of interest exists.

Submitted: October 3, 2016 Accepted: March 21, 2017 Published: May 4, 2017

Reference information: JCI Insight. 2017;2(9):e82922. https:// doi.org/10.1172/jii.insight.82922.

\section{Introduction}

Heart failure (HF) is a clinical syndrome of increasing incidence that affects multiple organ systems leading to metabolic and functional abnormalities (1). The normal adult heart utilizes fatty acids, glucose, amino acids, lactate, and ketone bodies for ATP production (2). Substrate preference depends on feeding and starvation cycles $(2,3)$, circadian rhythms (4), the functional state of the myocardium, and the impact of systemic metabolic derangements including diabetes mellitus and obesity (3, 5). Under physiological conditions, the adult heart preferentially uses fatty acids as fuel, while the failing myocardium increases the use of glucose for ATP production $(2,6,7)$. However, the failing myocardium develops a defect in use of both fatty acids and glucose associated with mitochondrial dysfunction, reduced oxidative metabolism, and accumulation of toxic lipid intermediates $(5,8,9)$. We and others have previously shown that ventricular remodeling in HF is associated with accumulation of potentially toxic lipids (8-13), inflammation (14), and apoptosis (15). However, the cause and consequences of these changes in lipid metabolism are still incompletely understood. One accumulated lipid is ceramide.

Increasing evidence supports a distinct role of ceramide signaling in cardiovascular disease states. Ceramides, a class of sphingolipids, are second messenger molecules that mediate multiple cellular functions (16). Ceramides are synthesized via several molecular pathways; however, how ceramide production is regulated in the normal and pathological myocardium is unclear. Understanding these 
pathways is especially important because changes in ceramide synthesis and accumulation may contribute to myocardial injury and the progression of $\operatorname{HF}(17,18)$.

Serine palmitoyl transferase (SPT), a heterodimer formed by 2 subunits, is the rate-limiting enzyme of de novo ceramide synthesis. Myriocin, an atypical amino acid derived from thermophilic fungi, is a pharmacologic inhibitor of SPT that competitively binds to the functional center of this enzyme complex. Most cells can also produce ceramide from sphingomyelin through acid sphingomyelinase (ASM); the heart is a major site of metabolism of circulating lipids within triglyceride-rich (TG-rich) lipoproteins that can supply substrates for generation of ceramides via this second pathway. Perhaps for this reason, myriocin treatment does not alter ceramide levels in normal hearts (17). Finally, ceramides can be synthesized from sphingosin-1-phosphate through the salvage pathway. Cardiomyocyte-specific deletion of SPT leads to a $\sim 35 \%$ reduction in heart ceramides, suggesting that in the stressed cellular environment - such as in inflammation and HF - the majority of ceramides seem to be produced by the de novo pathway of ceramide synthesis (19).

Ceramide signaling affects cell growth, apoptosis, and inflammation (16, 20, 21). In rat neonatal cardiomyocytes, C2 ceramide induces apoptotic cell death (20). Several interventions are known to cause ceramide accumulation, including prolonged oxidative stress of cultured cardiomyocytes and ischemia of isolated hearts $(18,21,22)$. Further, doxorubicin-induced cellular toxicity has been linked to increased ceramide synthesis, and inhibition of ceramide accumulation blocks the antineoplastic effects of this drug $(23,24)$. Doxorubicin-induced HF has been associated with ceramide accumulation and apoptosis $(20,25,26)$. Whether reduction of ceramide levels will improve late remodeling in $\mathrm{HF}$ has not been studied. We hypothesized that HF alters lipid metabolism, leading to altered cardiac ceramide content and composition, and that ceramide contributes to the progression of HF.

\section{Results}

Cardiac and circulating ceramide levels increase in human HF. Lipidomic analysis of samples obtained from patients with moderate and advanced HF were performed to analyze specific and total ceramides in both serum and myocardium. Patient characteristics and baseline laboratory data are summarized in Table 1. While levels of total circulating ceramides were not different between subjects with $\mathrm{HF}$ and controls (Figure 1A), several longand very long-chain ceramide species including C16, C18, C20:1, C20, C22:1, and C24:1 showed increased levels in serum of patients with HF (Figure 1B and Table 2). LDL and HDL cholesterol levels decreased with HF progression without differences in TG levels (Figure 1C). Data of various ceramide species in each patient subclassified by HF severity and controls are highlighted in Figure 1D. Tissue samples of myocardium obtained from patients with advanced $\mathrm{HF}$ at the time of cardiac surgery for ventricular assist device (VAD) placement, in comparison with samples obtained from nondiseased hearts, revealed increased total levels of cardiac ceramides (Figure 1E), as well as several long- and very long-chain ceramides including C16:1, C16, and C24:1 ceramides. Several other long- and very long-chain ceramides showed a trend toward increased levels in failing myocardium (C20:1, C20, C22:1, and C22; Figure 1, F and G, and Table 3).

The increased long-chain ceramides could be the product of several ceramide synthesis pathways The failing myocardium had increased levels of serine-palmitoyl transferase long chain 2 (SPTLC2) without significant changes in SPTLC1 and SPTLC3, suggesting an activation of the de novo pathway of cardiac ceramide synthesis (Figure 1, H and I). We did not find increased levels of ceramide synthetases, and in fact, protein levels of ceramide synthase 2 (CERS2) were decreased in failing myocardium. No changes were noted in other rate-limiting enzymes of cardiac ceramide synthesis including ASM, CERS1, and CERS5 (Figure 1I). Further, no differences in gene expression of any of the enzymes of ceramide synthesis were detectable between groups (data not shown).

Mechanical unloading decreases myocardial ceramides in human HF. Mechanical unloading through VAD placement is an established treatment option for patients with advanced HF and is associated with distinct effects of cardiac remodeling, function, and metabolism $(8,27,28)$. Myocardial samples obtained before and after VAD placement (patient characteristics summarized in Table 4) were analyzed by liquid chromatography-mass spectrometry (LS/MS) lipidomics. Myocardial mechanical unloading through VAD in patients with advanced HF resulted in a slight increase in circulating total levels of ceramides, including an increase in C14 and C24 ceramide (Figure 2, A-C, and Table 2). This might result from increased circulating lipoprotein levels associated with improved hepatic function following improved hemodynamics under VAD support (29). In contrast, total and individual ceramide species in the failing myocardium decreased 
Table 1. Demographic characteristics and baseline laboratory values of patients with HF and controls

\begin{tabular}{|c|c|c|c|}
\hline & Control $(n=22)$ & HF $(n=65)$ & $P$ value \\
\hline Male (n [\%]) & $12(55)$ & $58(89)$ & \\
\hline Age (yrs) & $53 \pm 10$ & $61 \pm 11$ & 0.003 \\
\hline $\mathrm{BMI}\left(\mathrm{kg} / \mathrm{m}^{2}\right)$ & $26 \pm 4$ & $27 \pm 5$ & 0.201 \\
\hline Heart rate & $77 \pm 9$ & $78 \pm 16$ & 0.952 \\
\hline Systolic BP (mmHg) & $123 \pm 8$ & $107 \pm 16$ & $<0.001$ \\
\hline Diastolic BP (mmHg) & $77 \pm 10$ & $69 \pm 12$ & 0.023 \\
\hline \multicolumn{4}{|l|}{ Etiology of HF (n [\%]) } \\
\hline Dilated Cardiomyopathy & - & $33(51)$ & \\
\hline Ischemic Cardiomyopathy & - & $11(17)$ & \\
\hline Other & - & $21(32)$ & \\
\hline Ejection fraction (\%) & $>55$ & $20 \pm 7$ & \\
\hline \multicolumn{4}{|l|}{ Medications } \\
\hline Diuretics & - & $46(71)$ & \\
\hline$\beta$-Blocker & - & $54(83)$ & \\
\hline ACE/ARBs & - & $45(69)$ & \\
\hline Aspirin & - & $36(55)$ & \\
\hline Coumadin & - & $19(29)$ & \\
\hline Statins & - & $40(62)$ & \\
\hline Insulin & - & $14(22)$ & \\
\hline Antiarrhythmic & - & $17(26)$ & \\
\hline Digoxin & - & $33(51)$ & \\
\hline Vasodilators & - & $2(3)$ & \\
\hline \multicolumn{4}{|l|}{ Laboratory Values } \\
\hline WBC $\left(1 \times 10^{9} / I\right)$ & $6.1 \pm 1.1$ & $7.3 \pm 2.8$ & 0.128 \\
\hline Hematocrit (\%) & $40 \pm 5$ & $34 \pm 9$ & 0.016 \\
\hline Platelets $\left(1 \times 10^{9} / \mathrm{I}\right)$ & $251 \pm 53$ & $204 \pm 72$ & 0.029 \\
\hline Glucose (mg/dl) & $87 \pm 11$ & $125 \pm 55$ & 0.004 \\
\hline Sodium (mEq/l) & $138 \pm 7$ & $136 \pm 4$ & 0.074 \\
\hline Potassium (mEq/l) & $4.5 \pm 0.4$ & $4.3 \pm 0.5$ & 0.129 \\
\hline BUN (mg/dl) & $15 \pm 4$ & $29 \pm 20$ & 0.004 \\
\hline Creatinine (mg/dl) & $0.94 \pm 0.24$ & $1.33 \pm 0.53$ & 0.003 \\
\hline Albumin (g/dl) & $4.5 \pm 0.3$ & $4.0 \pm 0.6$ & $<0.001$ \\
\hline Total bilirubin (mg/dl) & $0.54 \pm 0.17$ & $1.12 \pm 1.19$ & 0.038 \\
\hline Direct bilirubin (mg/dl) & $0.10 \pm 0.03$ & $0.52 \pm 1.52$ & 0.229 \\
\hline $\operatorname{AST}(\mathrm{U} / \mathrm{I})$ & $23 \pm 12$ & $29 \pm 22$ & 0.289 \\
\hline $\operatorname{ALT}(U / I)$ & $20 \pm 6$ & $25 \pm 17$ & 0.261 \\
\hline APT (U/I) & $67 \pm 17$ & $81 \pm 30$ & 0.070 \\
\hline $\mathrm{BNP}(\mathrm{pg} / \mathrm{ml})$ & - & $733 \pm 882$ & \\
\hline HbA1c (\%) & $5.3 \pm 0.2$ & $6.7 \pm 1.8$ & 0.087 \\
\hline Cholesterol (mg/dl) & $215 \pm 68$ & $149 \pm 46$ & $<0.001$ \\
\hline HDL (mg/dl) & $61 \pm 14$ & $41 \pm 18$ & 0.002 \\
\hline $\mathrm{LDL}(\mathrm{mg} / \mathrm{dl})$ & $110 \pm 46$ & $82 \pm 33$ & 0.016 \\
\hline Triglycerides (mg/dl) & $93 \pm 39$ & $142 \pm 76$ & 0.041 \\
\hline $\operatorname{TNF} \alpha(\mathrm{pg} / \mathrm{ml})$ & $1.95 \pm 0.62$ & $2.68 \pm 1.36$ & 0.044 \\
\hline
\end{tabular}

LVAD, left ventricular assist device; BMI, body mass index; BP, blood pressure; ACE/ARBs, Angiotensin-converting enzyme/Angiotensin II receptor blocker; BUN, blood urea nitrogen; AST, aspartate aminotransferase; ALT, alanine aminotransferase; APT, alkaline phosphatase; BNP, b-type natriuretic peptide; HbA1c, hemoglobin A1c; HDL, highdensity lipoprotein; LDL, low density lipoprotein; TNF $\alpha$, tumor necrosis factor $\alpha$. Continuous variables are expressed as mean \pm SD and categorical variables as number, \%.

following mechanical unloading through VAD placement, affecting nearly all chain lengths of ceramides (total ceramides as well as C16:1, C16, C20:1, C20, C22:1, and C24:1, with trends toward significance in C18:1 ceramide) as measured by LC/MS lipidomics in the human myocardial samples (Figure 2, D-F, and Table 3). Surprisingly, these reductions of ceramide levels were not accompanied by reduction in SPTLC2 
A

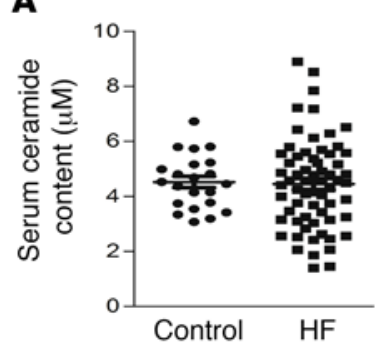

B

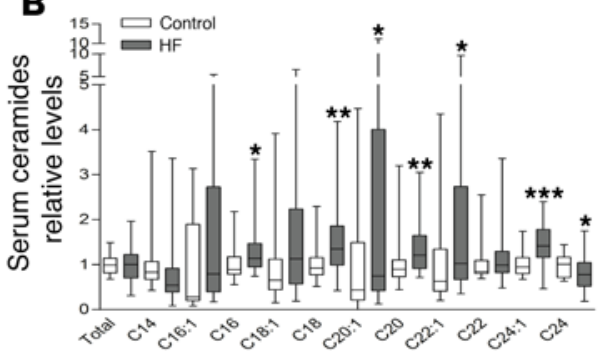

C

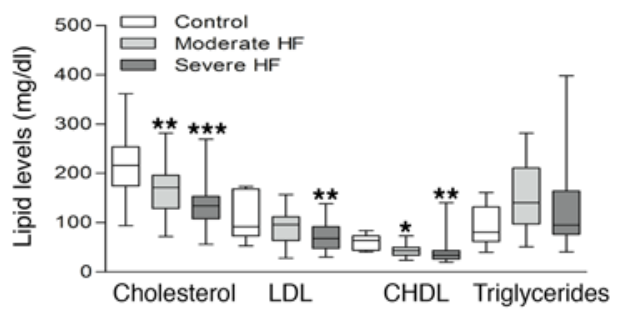

D

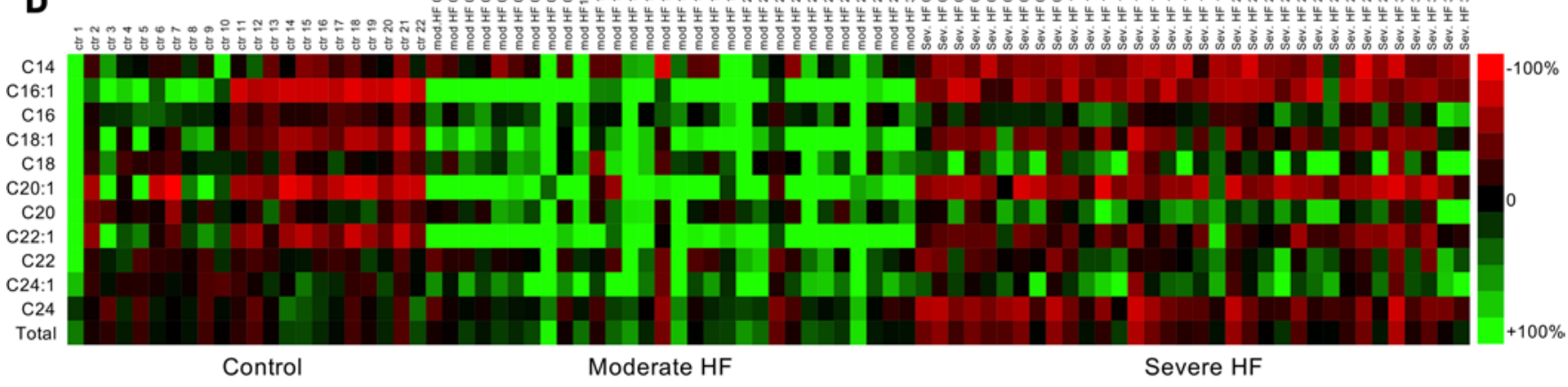

E

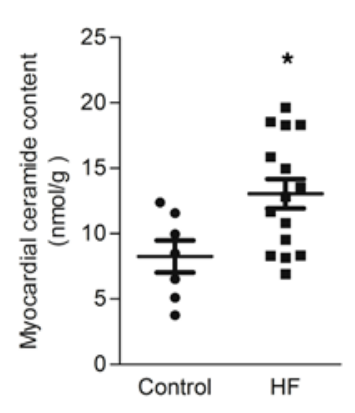

$\mathbf{F}$

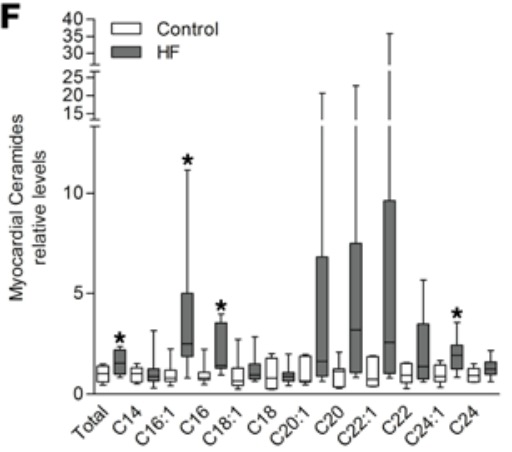

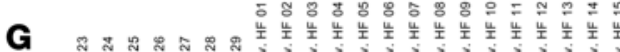

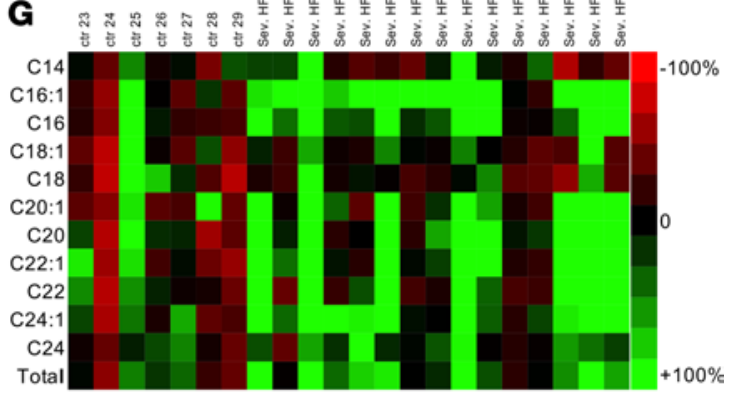

H

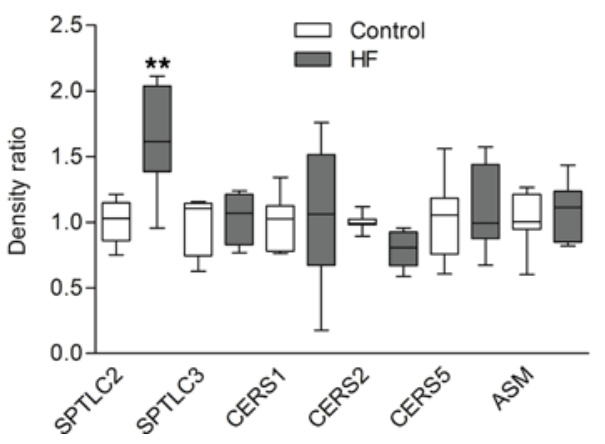

I

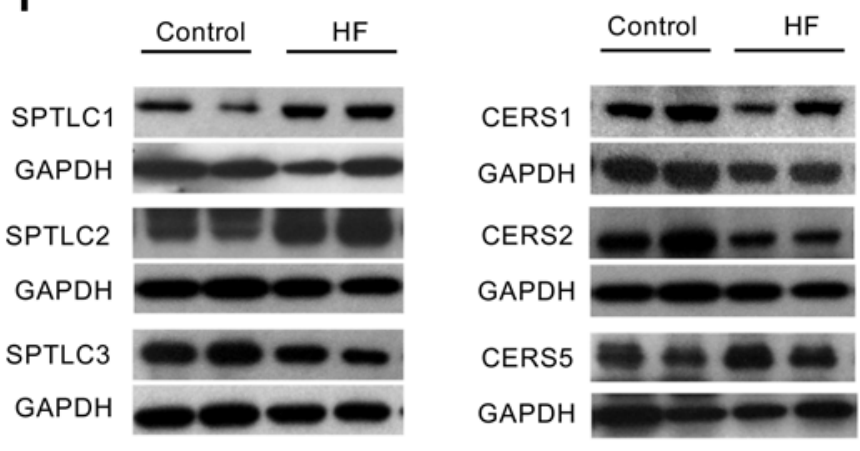

Figure 1. Analysis of circulating and myocardial ceramides in patients with HF. (A) Absolute circulating ceramide levels in patients with HF ( $n=64)$ and controls $(n=22)$. (B) Lipidomic analysis (LC/MS) of circulating ceramide species in patients with HF and controls. (C) Total cholesterol, LDL, and HDL cholesterol and triglyceride levels in patients with moderate $(n=30-31)$ and severe HF ( $n=28-32)$ and controls $(n=11-12)$. (D) Heatmap illustrating serum ceramide species in controls and patients with HF. Variabilities of ceramide levels are expressed in comparison with controls and blotted individually. (E) Absolute myocardial ceramide levels in patients with severe HF $(n=15)$ and controls $(n=7)$. (F) Myocardial ceramide species in patients with HF and controls. (C) Heatmap illustrating myocardial ceramide species normalized for controls. (H) Relative changes in key proteins of cellular ceramide synthesis $(n=4-7)$. (I) Western blot analysis of key proteins of ceramide synthesis pathways. Please note that some lanes of the HF group were used in Figure $2 \mathrm{H}$ to illustrate the HF Pre-VAD pattern. Two-tailed Student's $t$ test was used for 2 group comparisons, and one-way ANOVA was used for 3 group comparisons $\left({ }^{*} P<0.05,{ }^{* *} P<0.01,{ }^{* * *} P<0.001\right.$ versus control). 
Table 2. Absolute levels of circulating ceramide species in patients with HF before and after mechanical unloading through VAD implantation and controls

\begin{tabular}{|c|c|c|c|c|c|c|}
\hline & $\begin{array}{l}\text { Controls } \\
(n=22)\end{array}$ & $\begin{array}{l}\text { Heart Failure } \\
\text { All patients } \\
(n=64)\end{array}$ & $P$ value & $\begin{array}{c}\text { Heart Failure } \\
\text { pre-VAD } \\
(n=34)\end{array}$ & $\begin{array}{l}\text { Heart Failure } \\
\text { post-VAD } \\
(n=27)\end{array}$ & $P$ value \\
\hline C14 & $0.007 \pm 0.004$ & $0.005 \pm 0.004$ & 0.169 & $0.003 \pm 0.001$ & $0.004 \pm 0.002$ & 0.003 \\
\hline C16:1 & $0.038 \pm 0.037$ & $0.062 \pm 0.055$ & 0.067 & $0.018 \pm 0.009$ & $0.014 \pm 0.006$ & 0.076 \\
\hline C16 & $0.229 \pm 0.081$ & $0.287 \pm 0.100$ & 0.017 & $0.259 \pm 0.068$ & $0.258 \pm 0.077$ & 0.936 \\
\hline C18:1 & $0.026 \pm 0.024$ & $0.039 \pm 0.031$ & 0.072 & $0.018 \pm 0.008$ & $0.017 \pm 0.007$ & 0.554 \\
\hline C18 & $0.089 \pm 0.033$ & $0.132 \pm 0.063$ & 0.002 & $0.129 \pm 0.058$ & $0.124 \pm 0.051$ & 0.688 \\
\hline C20:1 & $0.003 \pm 0.003$ & $0.006 \pm 0.007$ & 0.038 & $0.001 \pm 0.001$ & $0.002 \pm 0.001$ & 0.73 \\
\hline C20 & $0.009 \pm 0.005$ & $0.013 \pm 0.005$ & 0.007 & $0.012 \pm 0.005$ & $0.013 \pm 0.005$ & 0.96 \\
\hline C22:1 & $0.022 \pm 0.021$ & $0.042 \pm 0.037$ & 0.018 & $0.015 \pm 0.006$ & $0.016 \pm 0.005$ & 0.544 \\
\hline$C 22$ & $0.203 \pm 0.079$ & $0.229 \pm 0.104$ & 0.265 & $0.210 \pm 0.072$ & $0.244 \pm 0.104$ & 0.144 \\
\hline C24:1 & $0.805 \pm 0.209$ & $1.178 \pm 0.374$ & 0.0001 & $1.069 \pm 0.361$ & $1.125 \pm 0.287$ & 0.517 \\
\hline C24 & $3.09 \pm 0.769$ & $2.454 \pm 1.067$ & 0.012 & $1.781 \pm 0.701$ & $2.556 \pm 1.191$ & 0.002 \\
\hline $\begin{array}{l}\text { Total } \\
\text { All cera }\end{array}$ & $\begin{array}{c}4.518 \pm 0.972 \\
\text { evels are express }\end{array}$ & $\begin{array}{l}4.447 \pm 1.634 \\
\text { n uM. }\end{array}$ & 0.848 & $3.519 \pm 1.135$ & $4.371 \pm 1.564$ & 0.017 \\
\hline
\end{tabular}

- rather, protein levels of SPTLC3 decreased in response to mechanical unloading (Figure 2, G and H). Further, protein levels of CERS2 decreased, while levels of CERS1, CERS5, and ASM did not show differences between pre- and post-VAD samples. No differences in gene expression of any enzymes in ceramide synthesis were detectable between groups (data not shown). These data suggest that myocardial unloading and the associated changes in reverse remodeling and metabolism are accompanied by reduction of some cardiac ceramide synthesis pathways.

Increased myocardial ceramide levels in an animal model of myocardial infarction. We next tested whether ischemia-induced HF in mice also have altered ceramide metabolism. Two weeks after induction of myocardial infarction (MI) through ligation of the left coronary artery, animals developed left ventricular dysfunction (fractional shortening [FS]: $17.4 \% \pm 1.8 \%$ in $\mathrm{HF}$ versus $36.1 \% \pm 1.9 \%$ in sham group, $P<0.001$ ) and progressive remodeling and dilation (left ventricular end-diastolic diameter [LVEDD]: $5.2 \pm 0.2 \mathrm{~mm}$ in $\mathrm{HF}$ versus $3.9 \pm 0.1 \mathrm{~mm}$ in the sham group, $P<0.001$ ).

$\mathrm{HF}$ also led to several changes in global metabolism. Mice with LV dysfunction had lower fasting glucose levels and increased circulating free fatty acid (FFA), total cholesterol (TC) levels, and unchanged TG levels 2 weeks after MI compared with sham surgery (glucose, $101.8 \pm 28.2$ versus $121.3 \pm 3.0 \mathrm{mg} / \mathrm{dl}, P=$ 0.2 ; FFA, $2.41 \pm 0.4$ versus $1.26 \pm 0.30 \mathrm{mmEq} / 1, P<0.01$; TC, $97.2 \pm 9.7$ versus $72.4 \pm 12.1 \mathrm{mg} / \mathrm{dl}, P<$ 0.05 ; TG, $59.1 \pm 6.1$ versus $53.4 \pm 12.2 \mathrm{mg} / \mathrm{dl}, P=\mathrm{NS}$; Table 5$)$. Total circulating ceramide levels $(+58 \% ; P$ $<0.05$ versus sham), as well as C16, C18, C24:1, C24, and C26:1, increased 2 weeks following MI (Figure $3 \mathrm{~A})$. This was accompanied by increased total myocardial ceramide levels 2 weeks after MI $(+31 \%, P<$ 0.05 versus sham). Lipidomic analysis of ceramide subspecies further revealed an increase in C14, C18, C20:1, C20, and C22:1 ceramides (Figure 3B and Table 6).

When the animals were followed longer (10 weeks) to monitor changes consistent with chronic left ventricular dysfunction, several of these parameters changed. Glucose and cholesterol levels returned to control levels, whereas TG and FFA levels increased in animals with LV dysfunction 10 weeks after MI compared with sham surgery (glucose, $138.4 \pm 7.1$ versus $131.0 \pm 4.5 \mathrm{mg} / \mathrm{dl}, P=\mathrm{NS}$; TG, $71.9 \pm 15.6$ versus $42.5 \pm 14.0 \mathrm{mg} / \mathrm{dl}, P<0.01 ; \mathrm{FFA}, 0.88 \pm 0.12$ versus $0.59 \pm 0.19 \mathrm{mEq} / 1, P<0.01$; TC, $96.5 \pm 30.9$ versus $100.9 \pm 7.6 \mathrm{mg} / \mathrm{dl}, P=\mathrm{NS}$; Table 5). While no differences in circulating total or individual ceramides were noted between the sham and MI group (except a trend toward higher C24 ceramide; Figure 3C), total cardiac ceramide content was increased 10 weeks after MI $(+19 \%, P<0.01$; Figure 3D and Table 7). No concomitant changes in other cardiac lipids such as TG or FFA were detectable (data not shown). Distinct ceramide subspecies including C16 ceramide $(+19 \%, P<0.05)$ and the very long-chain species C24:1 $(+44 \%, P<0.01)$ and $\mathrm{C} 24$ ceramide $(+39 \%, P<0.001)$ were increased in failing myocardium (Figure 3D).

Next, we analyzed the cardiac gene expression and protein levels of key enzymes in ceramide metabolism. We did not detect differences in cardiac gene expression of key enzymes of cellular ceramide syn- 
Table 3. Absolute levels of myocardial ceramide species in patients with advanced HF before and after mechanical unloading through VAD implantation and controls

\begin{tabular}{|c|c|c|c|c|c|}
\hline & $\begin{array}{c}\text { Controls } \\
(n=7)\end{array}$ & $\begin{array}{l}\text { Heart Failure } \\
\qquad(n=15)\end{array}$ & $P$ value & $\begin{array}{l}\text { Heart Failure } \\
\text { post-VAD } \\
(n=15)\end{array}$ & $P$ value $^{A}$ \\
\hline C14 & $87 \pm 30.87$ & $99.46 \pm 62.96$ & 0.628 & $87.27 \pm 36.47$ & 0.522 \\
\hline C16:1 & $31.11 \pm 18.99$ & $116 \pm 85.52$ & 0.018 & $40.95 \pm 21.72$ & 0.003 \\
\hline C16 & $919.5 \pm 527.7$ & $1,986 \pm 1027$ & 0.018 & $1,199 \pm 610.8$ & 0.016 \\
\hline C18:1 & $67.52 \pm 56.17$ & $77.21 \pm 38.13$ & 0.638 & $58.11 \pm 22.92$ & 0.108 \\
\hline C18 & $1,116 \pm 778.6$ & $1,106 \pm 480.3$ & 0.973 & $1,163 \pm 616.0$ & 0.779 \\
\hline C20:1 & $1.21 \pm 0.78$ & $5.5 \pm 6.5$ & 0.106 & $1.385 \pm 0.822$ & 0.023 \\
\hline C2O & $12.13 \pm 7.55$ & $60.98 \pm 70.41$ & 0.086 & $21.73 \pm 19.33$ & 0.047 \\
\hline C22:1 & $7.41 \pm 4.87$ & $51.73 \pm 68.68$ & 0.108 & $11.55 \pm 10.10$ & 0.033 \\
\hline C22 & $219.3 \pm 102.5$ & $508.8 \pm 389.3$ & 0.07 & $337.5 \pm 315.3$ & 0.196 \\
\hline C24:1 & $2,427 \pm 1,164$ & $4,657 \pm 1,855$ & 0.009 & $2,863 \pm 1,072$ & 0.003 \\
\hline C24 & $3,357 \pm 1,127$ & $4,379 \pm 1,401$ & 0.108 & $4,078 \pm 1,347$ & 0.554 \\
\hline $\begin{array}{l}\text { Total } \\
{ }^{A} \text { Comp }\end{array}$ & $\begin{array}{l}8,244 \pm 3,269 \\
\text { eefore VAD versu }\end{array}$ & $\begin{array}{l}13,048 \pm 4,368 \\
\text { r VAD implantation }\end{array}$ & $\begin{array}{l}0.018 \\
\text { amide le }\end{array}$ & $\begin{array}{l}9,862 \pm 3,345 \\
\text { xpressed in pmol }\end{array}$ & 0.033 \\
\hline
\end{tabular}

thesis. As noted in the tissues from humans with chronic HF, SPTLC2 protein levels were increased in the hearts of mice with LV dysfunction (SPTLC2: $+68 \%$ versus sham surgery; $P<0.05$ ) while protein levels of CERS2 and ASM did not change (Figure 3, E and F). No changes in gene or protein expression levels of ceramide synthesis-associated enzymes were detected at earlier time points.

SPT regulates cellular ceramide levels, apoptosis, and OXPHOS. SPTLC2 is only one subunit of the SPT complex and might not be rate-limiting for ceramide de novo synthesis. To test whether the isolated increase in SPTLC2 affects ceramide production, we tested the impact of SPTLC1and SPTLC2 overexpression on ceramide levels and composition in a human cardiomyocyte cell line (AC16). Expression plasmids for overexpression of human SPTLC1and SPTLC2 using pX3FLAG-CMV-7.X vectors were constructed and transfected into AC16 cells. Individual overexpression of both SPTLC1 or SPTLC2 resulted in robust accumulation of total C14, C16:1, C16, and C24:1 ceramide. Further, only SPTLC1 overexpression increased all cellular ceramides, including the very long-chain ceramides species C22:1, C24, C24:1, and C26:1 (Figure 4A). Overexpression of SPTLC1 and SPTLC2 was also associated with increased cellular apoptosis detected by annexin-V staining (Figure 4B). Further, analysis of cellular oxidative metabolism analyzed by Seahorse analysis showed inhibition of cellular oxidation with both SPTLC1and SPTLC2 overexpression (Figure 4, C and D). Finally, in order to test whether specific inhibition of SPT changes cellular ceramide levels, including specific very long-chain ceramides, we incubated AC16 cells with the specific SPT inhibitor myriocin. Incubation of AC16 cells with myriocin decreased total and C14, C18, C20:1, C22, C24:1, C24, C26:1, and C26 ceramides (Figure 4E).

Effects of SPT inhibition through myriocin on cardiac remodeling. We next tested whether myriocin-induced SPT inhibition altered cardiac ceramide metabolism and remodeling in the animal model of ischemic LV dysfunction. As above, MI was induced through ligation of the left coronary artery, and sham surgery was performed in the control cohort. MI-induced LV dysfunction was confirmed in all mice by echocardiography at 2 weeks after surgery, showing the expected decrease in LV function. Mice were randomized 2 weeks after MI to either a diet containing myriocin or normal chow diet for 8 weeks following MI. Serum glucose and lipid levels were analyzed at baseline, as well as 2 weeks and 8 weeks following MI, in both the myriocin and placebo treatment group. After 8 weeks on a myriocin-containing diet, there were no significant changes in serum glucose, TC, TG, and FFA levels compared with placebo (Table 5).

We next assessed whether myriocin treatment also prevented adverse cardiac effects due to MI. As noted above, infarcted mice had decreased FS and increased LVEDD in chow diet-fed mice 10 weeks after MI. LV dilation was prevented in animals in the myriocin group compared with the chow diet group (LVEDD, 4.8 \pm 0.3 versus $5.9 \pm 0.3 \mathrm{~mm}$ in placebo, $P<0.05$; Figure $5, \mathrm{~A}$ and $\mathrm{B}$ ). Further, myriocin-treated animals had better systolic function (FS, $19.8 \% \pm 2.4 \%$ versus $13.4 \% \pm 2.2 \%$ in placebo, $P<0.05$; Figure $5, \mathrm{C}$ and $\mathrm{D}$ ). No changes in LV function or structure were observed in the sham surgery group in either the myriocin or 
Table 4. Characteristics in patients with HF following mechanical unloading through VAD placement

\begin{tabular}{|c|c|c|c|}
\hline & $\begin{array}{l}\text { Pre-LVAD } \\
(n=34)\end{array}$ & $\begin{array}{c}\text { Post-LVAD } \\
(n=27)\end{array}$ & $P$ value \\
\hline \multicolumn{4}{|l|}{ Male (n [\%]) } \\
\hline Age (yrs) & $63 \pm 12$ & $63 \pm 10$ & 0.914 \\
\hline BMI $\left(\mathrm{kg} / \mathrm{m}^{2}\right)$ & $27 \pm 4$ & $26 \pm 3$ & 0.834 \\
\hline Heart Rate & $84 \pm 19$ & $84 \pm 13$ & 0.996 \\
\hline Systolic BP (mmHg) & $101 \pm 13$ & $109 \pm 18$ & 0.043 \\
\hline Diastolic BP (mmHg) & $65 \pm 12$ & $72 \pm 15$ & 0.048 \\
\hline \multicolumn{4}{|l|}{ Etiology of HF ( $n[\%])$} \\
\hline Ischemic cardiomyopathy & $20(59)$ & - & \\
\hline Dilated cardiomyopathy & $13(38)$ & - & \\
\hline Other & $1(3)$ & - & \\
\hline Ejection fraction (\%) & $18 \pm 5$ & $31 \pm 18$ & $<0.001$ \\
\hline \multicolumn{4}{|l|}{ Medications } \\
\hline Diuretics & $25(74)$ & $14(52)$ & \\
\hline$\beta$-Blocker & $26(76)$ & $20(74)$ & \\
\hline ACE/ARBs & $19(56)$ & $12(43)$ & \\
\hline Aspirin & $15(44)$ & $20(74)$ & \\
\hline Coumadin & $15(44)$ & $16(61)$ & \\
\hline Statins & $21(62)$ & $16(61)$ & \\
\hline Insulin & $9(26)$ & $5(17)$ & \\
\hline Antiarrhythmic & $13(38)$ & $6(22)$ & \\
\hline Digoxin & $17(50)$ & $5(17)$ & \\
\hline Vasodilators & $1(3)$ & $1(4)$ & \\
\hline \multicolumn{4}{|l|}{ Laboratory Values } \\
\hline WBC $\left(1 \times 10^{9} / \mathrm{I}\right)$ & $8.0 \pm 3.2$ & $8.3 \pm 3.3$ & 0.761 \\
\hline Hematocrit (\%) & $30 \pm 10$ & $33 \pm 5$ & 0.086 \\
\hline Platelets $\left(1 \times 10^{9} / \mathrm{I}\right)$ & $201 \pm 85$ & $199 \pm 79$ & 0.927 \\
\hline Glucose (mg/dl) & $137 \pm 61$ & $126 \pm 61$ & 0.541 \\
\hline Sodium (mEq/l) & $135 \pm 4$ & $138 \pm 4$ & 0.002 \\
\hline Potassium (mEq/l) & $4.2 \pm 0.6$ & $4.2 \pm 0.6$ & 0.878 \\
\hline BUN (mg/dl) & $38 \pm 25$ & $29 \pm 14$ & 0.123 \\
\hline Creatinine (mg/dl) & $1.54 \pm 0.61$ & $1.37 \pm 0.67$ & 0.307 \\
\hline Albumin (g/dl) & $3.6 \pm 0.5$ & $3.8 \pm 0.5$ & 0.054 \\
\hline Total bilirubin (mg/dl) & $1.43 \pm 1.56$ & $1.03 \pm 0.81$ & 0.218 \\
\hline Direct bilirubin (mg/dl) & $0.54 \pm 0.84$ & $0.37 \pm 0.47$ & 0.367 \\
\hline AST (U/I) & $33 \pm 28$ & $52 \pm 57$ & 0.104 \\
\hline $\operatorname{ALT}(U / I)$ & $27 \pm 22$ & $26 \pm 26$ & 0.864 \\
\hline APT (U/I) & $81 \pm 31$ & $77 \pm 25$ & 0.586 \\
\hline BNP (pg/ml) & $1,040 \pm 935$ & $676 \pm 443$ & 0.074 \\
\hline HbA1c (\%) & $6.86 \pm 2.03$ & $6.15 \pm 1.60$ & 0.232 \\
\hline Cholesterol (mg/dl) & $137 \pm 41$ & $163 \pm 61$ & 0.053 \\
\hline HDL (mg/dl) & $38 \pm 23$ & $41 \pm 13$ & 0.681 \\
\hline LDL (mg/dl) & $72 \pm 28$ & $99 \pm 59$ & 0.041 \\
\hline Triglycerides (mg/dl) & $135 \pm 85$ & $156 \pm 70$ & 0.343 \\
\hline \multicolumn{4}{|c|}{$\begin{array}{l}\text { LVAD, left ventricular assist device; BMI, body mass index; BP, blood pressure; ACE/ARBs, Angiotensin-converting } \\
\text { enzyme/Angiotensin II receptor blocker; BUN, blood urea nitrogen; AST, aspartate aminotransferase; ALT, alanine } \\
\text { aminotransferase; APT, alkaline phosphatase; BNP, b-type natriuretic peptide; HbA1c, hemoglobin A1c; HDL, high- } \\
\text { density lipoprotein; LDL, low-density lipoprotein; TNF } \alpha \text {, tumor necrosis factor } \alpha \text {. Continuous variables are expressed as } \\
\text { mean } \pm \text { SD and categorical variables as number, \%. }\end{array}$} \\
\hline
\end{tabular}

the chow control diet group (Figure 5, A and C). Compared with sham chow and sham myriocin groups, the heart weight over body weight $(\mathrm{HW} / \mathrm{BW})$ ratio increased significantly in the HF chow group but not in the $\mathrm{HF}$ myriocin group (HF chow, $0.55 \pm 0.08$, versus sham chow, $0.47 \pm 0.02, P<0.05$; Table 5). Body weight was lower in the myriocin diet group compared with animals with $\mathrm{HF}$ on chow $\operatorname{diet}(P<0.001$; Table 5$)$. 

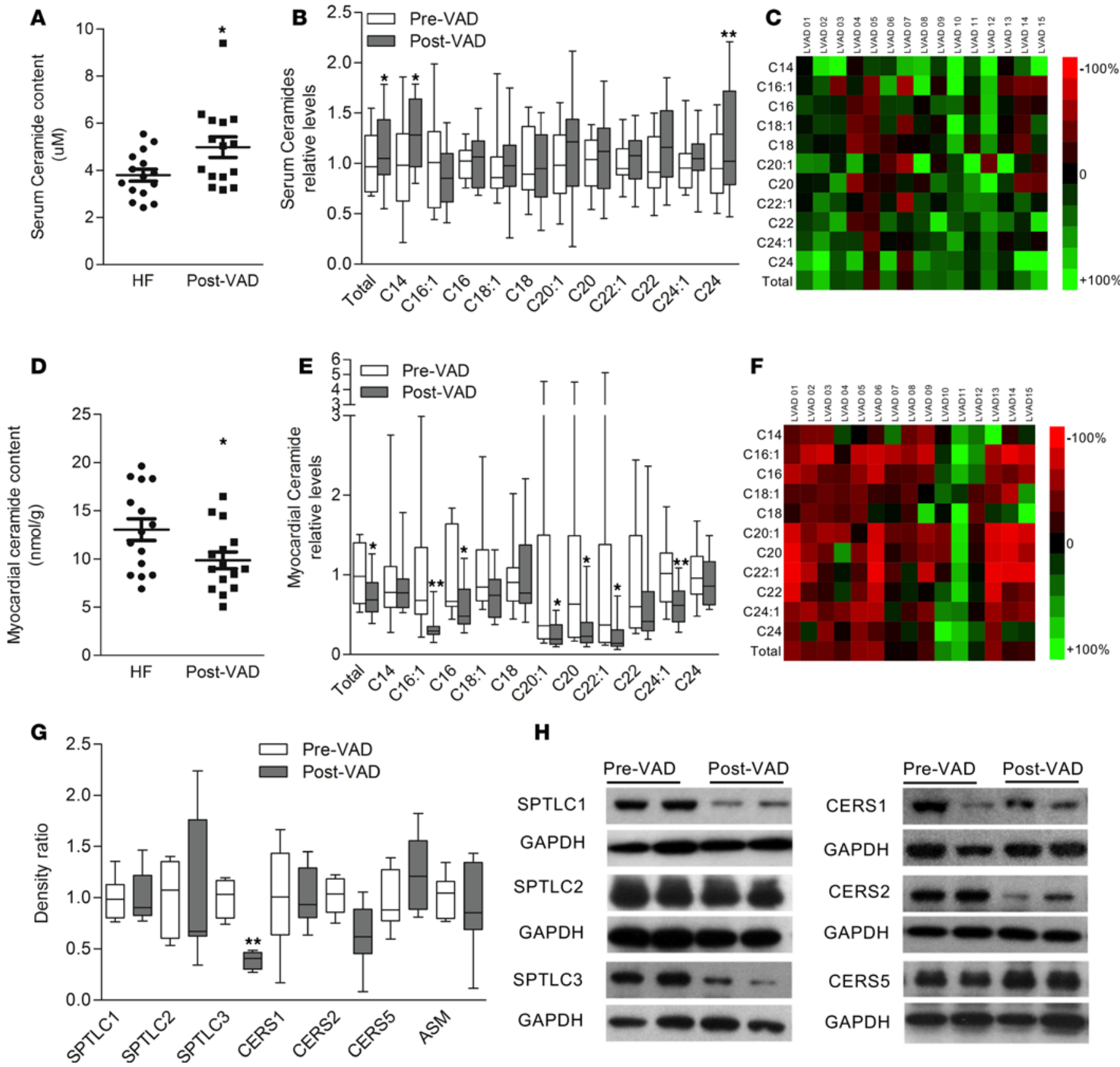

Figure 2. Mechanical unloading decreases circulating and myocardial ceramides in patients with HF. (A) Absolute circulating ceramide levels in patients with advanced HF before $(n=15)$ and after $(n=15)$ VAD implantation $(n=15$ paired samples). (B) Relative differences in circulating ceramide species in patients with advanced HF before and after VAD implantation ( $n=15$, paired). (C) Heatmap illustrating dynamics in total and individual serum ceramide levels (expressed as ratio of levels at the time of explantation versus implantation of VAD). (D) Absolute myocardial ceramide levels in patients at the time of VAD implantation ( $n=15)$ compared with VAD explantation $(n=15)$. (E) Dynamics in myocardial ceramide species levels during the time of VAD support (analysis before versus after VAD) $(n=15)$. (F) Heatmap illustrating dynamics in myocardial ceramide species (expressed as ratio of levels at the time of explantation compared with implantation). (C) Relative differences in key proteins of ceramide synthesis $(n=4-7)$. (H) Western blot analysis of key proteins of various ceramide synthesis pathways. Please note that some lanes of the HF Pre-VAD group were used in Figure 1 to illustrate the HF pattern. VAD, ventricular assist device. Two-tailed Student's $t$ test was used for analyzing data ( ${ }^{*} P<0.05,{ }^{* *} P<0.01$ versus Pre-VAD).

Inhibition of SPT through myriocin treatment reduces cardiac inflammation and fibrosis. Quantification of myocardial collagen content using Masson's trichrome staining showed that collagen after MI was 10 -fold greater in control than in myriocin-treated mouse hearts $(0.08 \% \pm 0.04 \%$ in myriocin versus $0.7 \% \pm 0.3 \%$ brightfield image in placebo, $P<0.05)$. Cardiac macrophage content assessed by IHC increased after MI, but myriocin decreased macrophage content compared with placebo $(34 \pm 5.3$ in 
Table 5. Group Characteristics and systemic metabolic markers of glucose and lipid metabolism

\begin{tabular}{|c|c|c|c|c|}
\hline & \multicolumn{2}{|c|}{ Sham } & \multicolumn{2}{|c|}{ Ischemic LV Dysfunction } \\
\hline & $\begin{array}{l}\text { Chow } \\
(n=20)\end{array}$ & $\begin{array}{c}\text { Myriocin } \\
(n=7)\end{array}$ & $\begin{array}{l}\text { Chow } \\
(n=24)\end{array}$ & $\begin{array}{c}\text { Myriocin } \\
(n=18)\end{array}$ \\
\hline \multicolumn{5}{|l|}{$B W(g)$} \\
\hline 2 wks post-MI & $26.12 \pm 1.3$ & $28.17 \pm 1.3$ & $28.2 \pm 2.5$ & $27.33 \pm 1.5$ \\
\hline 10 wks post-MI & $28.3 \pm 2.4$ & $25.9 \pm 2.7$ & $30.7 \pm 3.0^{A}$ & $27.0 \pm 1.5^{\mathrm{B}}$ \\
\hline \multicolumn{5}{|l|}{ HW/BW(\%) } \\
\hline 10 wks post-Ml & $0.47 \pm 0.02$ & $0.48 \pm 0.05$ & $0.55 \pm 0.08^{c}$ & $0.53 \pm 0.07$ \\
\hline \multicolumn{5}{|l|}{ Glucose (mg/dl) } \\
\hline Baseline & $148.6 \pm 14.5$ & $142.0 \pm 11.3$ & $138.8 \pm 12.5$ & $144.5 \pm 5.8$ \\
\hline 2 wks post-MI & $121.3 \pm 3.0$ & $125.8 \pm 5.0$ & $101.8 \pm 28.2$ & $126.0 \pm 19.5$ \\
\hline 10 wks post-Ml & $131.0 \pm 4.5$ & $123.5 \pm 5$ & $138.4 \pm 7.1$ & $132.2 \pm 12.7$ \\
\hline \multicolumn{5}{|c|}{ Triglycerides (mg/dl) } \\
\hline Baseline & $63.6 \pm 10.7$ & $60.2 \pm 15.4$ & $67.0 \pm 7.8$ & $60.2 \pm 5.8$ \\
\hline 2 wks post-MI & $53.4 \pm 12.2$ & $56.95 \pm 8.9$ & $59.1 \pm 6.1$ & $48.7 \pm 17.9$ \\
\hline 10 wks post-MI & $42.5 \pm 14.0$ & $33.1 \pm 4.5$ & $71.9 \pm 15.6^{c}$ & $61.2 \pm 17.1^{\ominus}$ \\
\hline \multicolumn{5}{|l|}{ Cholesterol (mg/dl) } \\
\hline Baseline & $82.6 \pm 9.4$ & $84.7 \pm 10.9$ & $84.4 \pm 7.5$ & $79.4 \pm 10.9$ \\
\hline 2 wks post-MI & $72.4 \pm 12.1$ & $82.0 \pm 15.7$ & $97.2 \pm 9.7^{E}$ & $83.9 \pm 6.6$ \\
\hline 10 wks post-MI & $100.9 \pm 7.6$ & $118.3 \pm 21.8$ & $96.5 \pm 30.9$ & $97.0 \pm 34.7$ \\
\hline \multicolumn{5}{|l|}{ FFA (mEq/l) } \\
\hline Baseline & $0.6 \pm 0.17$ & $0.6 \pm 0.13$ & $0.69 \pm 0.12$ & $0.66 \pm 0.18$ \\
\hline 2 wks post-MI & $1.26 \pm 0.30$ & $0.93 \pm 0.21$ & $2.4 \pm 0.40^{F}$ & $1.82 \pm 0.56^{D}$ \\
\hline \multicolumn{5}{|c|}{$\begin{array}{l}\text { Animal model of myocardial ischemia compared with sham surgery and treatment effects of myriocin compared with } \\
\text { placebo. }{ }^{A} P<0.001 \text { compared with sham myriocin, }{ }^{\mathrm{B}} P<0.001 \text { compared with } \mathrm{LV} \text { dysfunction chow, }{ }^{\mathrm{C}} P<0.05 \text { compared } \\
\text { with sham chow and myriocin, }{ }^{\mathrm{D}} P<0.05 \text { compared with sham myriocin, }{ }^{\mathrm{E}} P<0.05 \text { compared with sham chow, }{ }^{\mathrm{F}} P<0.01 \\
\text { compared with sham chow and myriocin. } \mathrm{HW} \text {, heart weight; BW, body weight. HW/BW assessed at the time of sacrifice } \\
\text { at } 10 \text { weeks post-MI. }\end{array}$} \\
\hline
\end{tabular}

myriocin versus $59 \pm 8.1$ cells per brightfield in placebo, $P<0.05)$. Myocardial apoptosis detected by the TUNEL staining increased after MI and showed a trend toward a reduction under myriocin treatment $(P<0.1$; Figure 5, E-G). No significant differences were noted in the expression of several key apoptotic genes, including $\mathrm{Bcl} 2$, Bcl-XL, CHOP, and Bax, which only showed a trend toward lower levels in the myriocin group after MI (data not shown).

Effects of SPT inhibition through myriocin on cardiac ceramide synthesis and composition. We next analyzed the myocardial lipid composition by LC/MS/MS lipidomics. Treatment with myriocin decreased several ceramide species in the failing myocardium $(\mathrm{C} 16,-19 \%$; $\mathrm{C} 18: 1,-22 \%$; $222,-39 \%$; C24:1, -20\%; C24, -32\%; C26:1, $-43 \%$; and C26, $-40 \%$; all $P<0.05$ versus placebo; $n=9$ per group) (Figure $5 \mathrm{H}$ ) but did not affect circulating levels of ceramides. Only C18 ceramide, a selective product of CerS1 (30) was significantly increased in the myriocin treatment group, consistent with increased protein levels of CerS1. Myriocin treatment after MI did not change cardiac TG levels $(3.47 \pm 0.45$ versus $3.23 \pm 0.89 \mathrm{mg} / \mathrm{g}, P=\mathrm{NS}$; data not shown). Further, no changes in serum FFAs and TC were noted compared with controls on chow diet (FFA, $0.85 \pm 0.24$ versus $0.88 \pm 0.12 \mathrm{mEq} / 1$ in controls, $P$ $=\mathrm{NS}$; TC, $97.0 \pm 34.7$ versus $96.5 \pm 30.9 \mathrm{mg} / \mathrm{dl}$ in controls, $P=\mathrm{NS}$; Table 5$)$.

Preservation of cardiac function in Sptlc2 gene-deletion mice following MI. Cardiomyocyte-specific gene deletion of Sptlc2 mice were created and tested compared with floxed control mice. Lipidomic analysis of total and individual ceramides in the myocardium of Sptlc2-KO mice showed significant decrease when compared with floxed mice. (Total: $-31 \%, P<0.05$ versus floxed, $n=6$; Figure $5 \mathrm{I}$ ). We tested LV function at 2 weeks and at 10 weeks after MI, as well as subsequent development of ischemic cardiomyopathy in Sptlc2 $2^{-1-}$ and floxed mice. Both groups of mice developed acute cardiac dysfunction at 2 weeks following MI (FS, $18.2 \% \pm 3.3 \%$ in Sptlc $2^{--}$versus $21.2 \% \pm 4 \%$ in controls, no significance;

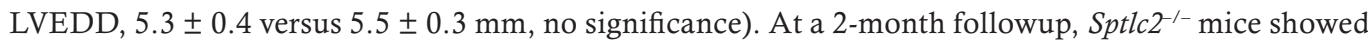



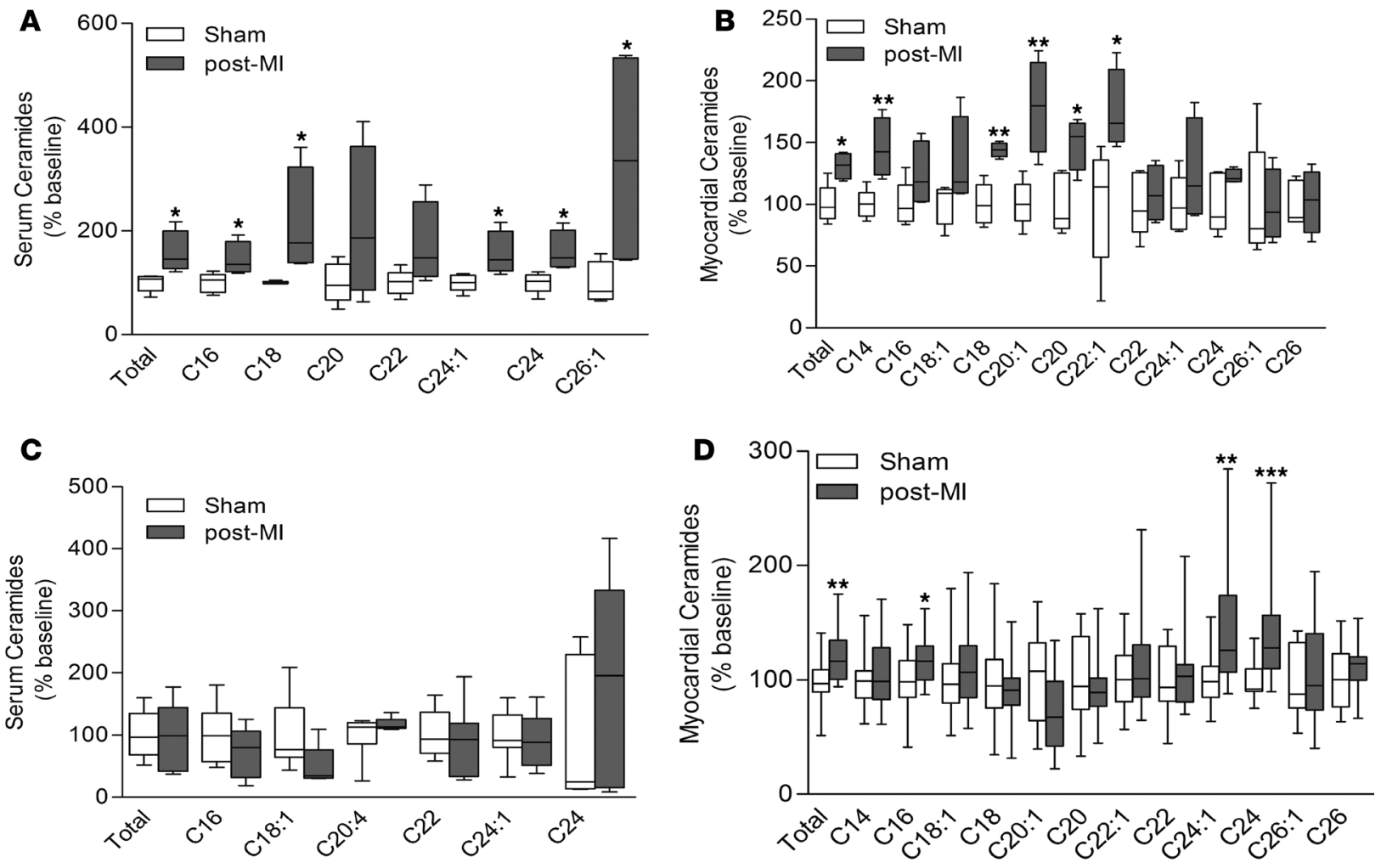

$\mathbf{E}$

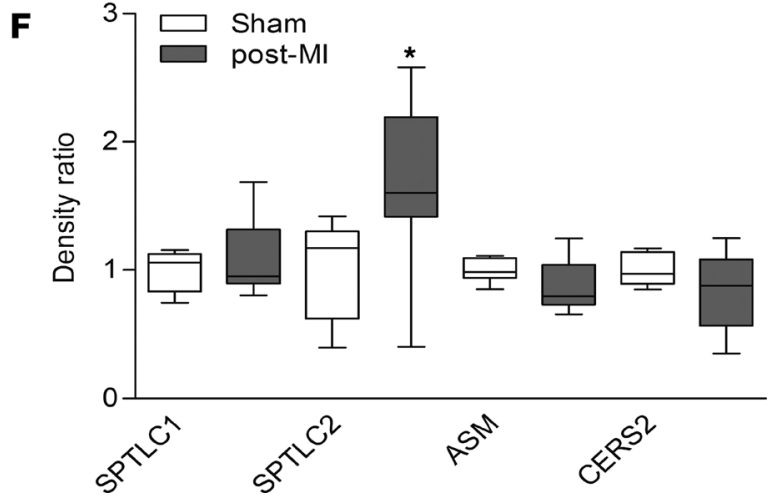

Figure 3. Increased levels of circulating and myocardial ceramides $\mathbf{2}$ weeks and $\mathbf{1 0}$ weeks following MI. (A) Increased serum levels of total ceramides and various ceramide subspecies 2 weeks after MI $(n=4-5)$. (B) Increased myocardial levels of total ceramides and various ceramide subspecies 2 weeks after MI ( $n$ $=4-5$ ). (C) Lipidomic analysis of ceramide subspecies showing profiles of circulating levels of ceramides in animals 10 weeks after MI ( $n=10-12)$. (D) Lipidomic analysis of myocardial ceramides 10 weeks after MI $(n=20)$. (E) Western blot analysis of key enzymes of cellular ceramide synthesis $(n=6-9)$. (F) Quantitative analysis of key enzymes of ceramide synthesis ( $n=6-9$ per group). Two-tailed Student's $t$ test was used ( $P<0.05,{ }^{* *} P<0.01,{ }^{* *} P<0.001$ versus sham).

less impairment in LV systolic function compared with control mice $(-9 \% \pm 11.5 \%$ versus $-38 \% \pm$

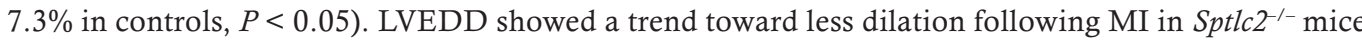
compared with controls (LVEDD, $+5.5 \% \pm 2.9 \%$ versus $+17.1 \% \pm 4.3 \%, P=0.08$; Figure $5, \mathrm{~J}$ and $\mathrm{K}$ ).

\section{Discussion}

Ceramides are a family of sphingosine species defined by the length and saturation of acyl chains. Increasing evidence suggests a role of ceramide species in cellular signaling $(16,24)$, metabolism $(16)$, and life span $(18,23)$. Total ceramides accumulate in the myocardium during ischemia and reperfusion, in animal models of HF, and in human cardiomyopathies (8). The biochemical pathways responsible for greater ceramide synthesis in $\mathrm{HF}$ and the specific ceramide species and their chain lengths and their distinct specific pathophysiological effects on cardiac function in nondiseased and failing hearts are still incompletely understood. 
Table 6. Absolute levels of circulating ceramide species in mice with HF 2 weeks after MI

\begin{tabular}{|c|c|c|c|c|c|c|}
\hline & \multicolumn{3}{|c|}{ Serum (nM) } & \multicolumn{3}{|c|}{ Myocardium (nmol/g) } \\
\hline & $\begin{array}{l}\text { Sham } \\
(n=5)\end{array}$ & $\begin{array}{l}\text { Heart Failure } \\
\qquad(n=4)\end{array}$ & $P$ value & $\begin{array}{l}\text { Sham } \\
(n=5)\end{array}$ & $\begin{array}{l}\text { Heart Failure } \\
\quad(n=4)\end{array}$ & $P$ value \\
\hline C14 & & & & $0.050 \pm 0.002$ & $0.073 \pm 0.006$ & 0.007 \\
\hline C16 & $159.6 \pm 13.2$ & $232.1 \pm 26.01$ & 0.033 & $6.187 \pm 0.501$ & $7.675 \pm 0.809$ & 0.146 \\
\hline C18:1 & & & & $0.303 \pm 0.022$ & $0.402 \pm 0.055$ & 0.112 \\
\hline C18 & $6.688 \pm 0,082$ & $14.23 \pm 3.479$ & 0.043 & $5.595 \pm 0.415$ & $8.052 \pm 0.162$ & 0.002 \\
\hline C2O:1 & & & & $0.037 \pm 0.003$ & $0.066 \pm 0.007$ & 0.005 \\
\hline C2O & $0.706 \pm 0.121$ & $1.495 \pm 0.521$ & 0.142 & $0.051 \pm 0.005$ & $0.076 \pm 0.005$ & 0.014 \\
\hline C22:1 & & & & $0.018 \pm 0.003$ & $0.032 \pm 0.003$ & 0.033 \\
\hline C22 & $73.34 \pm 7.911$ & $126.5 \pm 29.66$ & 0.095 & $0.107 \pm 0.012$ & $0.116 \pm 0.012$ & 0.625 \\
\hline C24:1 & $404.2 \pm 29.49$ & $627.8 \pm 86.61$ & 0.031 & $0.683 \pm 0.07$ & $0.860 \pm 0.144$ & 0.278 \\
\hline C24 & $471.3 \pm 40.82$ & $755.0 \pm 92.08$ & 0.018 & $1.781 \pm 0.701$ & $2.556 \pm 1.191$ & 0.002 \\
\hline C26:1 & $1.181 \pm 0.206$ & $3.994 \pm 1.300$ & 0.047 & $0.190 \pm 0.040$ & $0.187 \pm 0.027$ & 0.949 \\
\hline C26 & & & & $0.062 \pm 0.005$ & $0.063 \pm 0.008$ & 0.877 \\
\hline Total & $1,117 \pm 83.97$ & $1,761 \pm 231.8$ & 0.024 & $15.35 \pm 1.069$ & $20.14 \pm 0.821$ & 0.011 \\
\hline
\end{tabular}

Our current study reveals that ceramide metabolism is altered in the failing myocardium both in humans with $\mathrm{HF}$ and in animals with ischemic cardiomyopathy. Patients with various stages of HF show increased circulating levels of ceramides, as well as elevated cardiac levels of ceramides, particularly long- and very longchain species. Abnormal ceramide metabolism and accumulation of ceramides respond to mechanical unloading in humans following VAD implantation, while circulating levels appear not to be affected. We reproduced these alterations in ceramide accumulation in a rodent model of ischemic left ventricular dysfunction. Ten weeks after MI, cardiac ceramides increased with a specific increase in C22, C24, and C26 ceramides.

Our current study further explored the pathways associated with greater ceramide production. Both mice and humans with HF had increased levels of SPTLC2, a subunit of the SPT de novo synthesis pathway rate-limiting enzyme. Protein levels of SPTLC1, the other member of this complex, were not significantly changed. Neither ceramide synthetases nor sphingomyelinase were altered by HF.

To understand the specific role of ceramide synthesis, in particular the de novo pathway, we utilized cellular overexpression of both SPTLC1 and SPTLC2. We used this overexpression approach since the cellular uptake of ceramides with chain lengths longer than C8 is ineffective. Prior studies, therefore, have used short-chain ceramides C2-C6 to study ceramide-specific effects (18). These short-chain species are less relevant in regards to biologic effects and total amounts in cells and the myocardium where most common species are C16, C22, C24 and C24:1 ceramides (see lipidomic data in Tables 2 and 3). Our overexpression approach led to a robust increase in total ceramides and C14-C26:1 species with slightly higher levels under SPTLC1 overexpression compared with SPTLC2 overexpression. These changes were accompanied by increased apoptosis in AC16 cells. Further, our studies revealed profound effects of SPTLC1 and SPTLC2 overexpression on cellular oxidative metabolism. Both basal respiration rates and total respiratory capacity were decreased in SPTLC1- and SPTLC2-overexpressing cells compared with controls. These findings suggest a distinct role of increased cellular ceramide levels on both cellular viability and oxidative metabolism. Thus, the data point to increased de novo synthesis as a likely cause of ceramide accumulation, and we tested this by blocking the de novo synthesis pathway.

We used myriocin, a pharmacologic inhibitor of the SPT enzyme complex, in both the animal model of ischemic cardiomyopathy and in AC 16 cells in vitro. Prior studies have used this compound in animal models of atherosclerosis (31), hyperlipidemia (32), and lipotoxic cardiomyopathy (17). We observed a striking preservation of ventricular size and mass, as well as FS and LVEDD in the myriocin-treated group of animals after MI. This was accompanied by reduced myocardial macrophage infiltration and cardiac fibrosis, both hallmarks of cardiac remodeling in advanced ventricular dysfunction. Consistent with our findings, systolic function in mice with cardiomyocyte expression of anchored lipoprotein lipase improved in response to myriocin treatment (17). Our study revealed a mild decrease in overall body weight under myriocin treatment compared with controls in both the 
Table 7. Absolute levels of circulating ceramide species in mice with HF 10 weeks after MI

\begin{tabular}{|c|c|c|c|c|c|c|}
\hline & \multicolumn{3}{|c|}{ Serum (nM) } & \multicolumn{3}{|c|}{ Myocardium (nmol/g) } \\
\hline & $\begin{array}{l}\text { Sham } \\
(n=10)\end{array}$ & $\begin{array}{l}\text { Heart Failure } \\
\qquad(n=12)\end{array}$ & $P$ value & $\begin{array}{c}\text { Sham } \\
(n=20)\end{array}$ & $\begin{array}{l}\text { Heart Failure } \\
\qquad(n=20)\end{array}$ & P value \\
\hline C14 & & & & $0.033 \pm 0.003$ & $0.036 \pm 0.003$ & 0.466 \\
\hline C16 & $146.2 \pm 21.77$ & $109.3 \pm 16.26$ & 0.1811 & $4.488 \pm 0.369$ & $5.710 \pm 0.392$ & 0.029 \\
\hline C18:1 & $133.3 \pm 32.14$ & $66.46 \pm 20.13$ & 0.128 & $0.277 \pm 0.059$ & $0.343 \pm 0.069$ & 0.468 \\
\hline C18 & & & & $3.126 \pm 0.528$ & $2.753 \pm 0.407$ & 0.579 \\
\hline C20:1 & & & & $0.262 \pm 0.082$ & $0.205 \pm 0.080$ & 0.626 \\
\hline C20:4 & $42.56 \pm 6.359$ & $49.82 \pm 2.075$ & 0.344 & & & \\
\hline C20 & & & & $0.085 \pm 0.008$ & $0.074 \pm 0.007$ & 0.314 \\
\hline C22:1 & & & & $0.077 \pm 0.018$ & $0.099 \pm 0.032$ & 0.547 \\
\hline C22 & $22.39 \pm 2.473$ & $20.54 \pm 3.560$ & 0.686 & $0.122 \pm 0.008$ & $0.129 \pm 0.012$ & 0.314 \\
\hline C24:1 & $153.00 \pm 18.03$ & $140.00 \pm 18.23$ & 0.621 & $1.057 \pm 0.100$ & $1.600 \pm 0.242$ & 0.044 \\
\hline C24 & $132.90 \pm 45.72$ & $244.8 \pm 62.03$ & 0.177 & $1.319 \pm 0.211$ & $1.757 \pm 0.231$ & 0.171 \\
\hline C26:1 & & & & $0.167 \pm 0.018$ & $0.178 \pm 0.026$ & 0.718 \\
\hline C26 & & & & $0.041 \pm 0.004$ & $0.046 \pm 0.005$ & 0.498 \\
\hline Total & $577.8 \pm 66.42$ & $575.4 \pm 84.83$ & 0.983 & $10.85 \pm 0.908$ & $12.63 \pm 0.541$ & 0.100 \\
\hline
\end{tabular}

sham and the MI groups, but no differences in mortality between the groups. Of note, we did not observe changes in TC, but we observed a distinct increase in TG and FFAs following MI. Increased levels of TG decreased in response to myriocin treatment but did not reach levels of the sham group on chow diet. In our study, serum glucose and the glucose tolerance test did not show differences at baseline and in response to myriocin treatment. For further confirmation, ischemic cardiomyopathy was induced in Sptlc2-KO mice, and we found preservation of cardiac function and reduced remodeling in Sptlc2-KO mice compared with controls.

A major advantage of our current study is the translational analysis of samples from animal models of $\mathrm{HF}$, as well as tissue and serum obtained from patients with advanced cardiomyopathies. Further, we could test the reversibility of changes in ceramide synthesis and levels after mechanical unloading through the implantation of VADs in humans. Finally, we are the first to our knowledge to report clusters of longand very long-chain ceramides both in serum and myocardium analyzed by LC/MS lipidomics that are distinctively different in both human and rodent failing myocardium. Our findings show that mechanical unloading reverses abnormal ceramide synthesis, changes protein levels of key enzymes of ceramides synthesis pathways, and partially normalizes cardiac ceramide levels and composition. These data are in line with the known decrease in myocardial inflammation (33), improved metabolism (8), and correction in extracellular matrix turnover (27) following VAD placement.

Of interest, it is well known that HF leads to reduced fatty acid oxidation, and it appears that along with this, a greater proportion of cardiac fatty acids are shunted to the ceramide synthesis pathway. Inhibition of the SPT enzyme complex through myriocin inhibits ceramide accumulation with reduction in very long-chain ceramide species; total ceramide content shows less change, as the failing myocardium might revert to greater use of ceramidases. It was unclear from our in vivo studies whether this change in ceramide production was due to a direct effect of myriocin or because myriocin treatment prevented the progressive LV remodeling following MI. For this reason, we studied the effects of myriocin on cardiomyocyte-like cells, which confirmed its distinct effects on reducing ceramide synthesis and levels, including a reduction of very long-chain ceramides.

Ceramides are synthesized through 3 major pathways: the de novo pathway, which generates ceramides from serine and palmitoyl-CoA; the sphingomyelinase pathway via degradation of sphingomyelin; and the salvage pathway through generation from glycosphingolipids (16). The de novo pathway of ceramide synthesis produces around $30 \%$ of total cellular ceramides under basal conditions in the heart (19) and is induced in inflammation and hypoxia (16). Several recent studies have reported specific biological effects of distinct ceramides defined by the fatty acid chain length $(34,35)$. It has been postulated that very long-chain ceramides (C24-C26) are more cytotoxic and shorter chain (C20-C22) ceramides may have a 
A

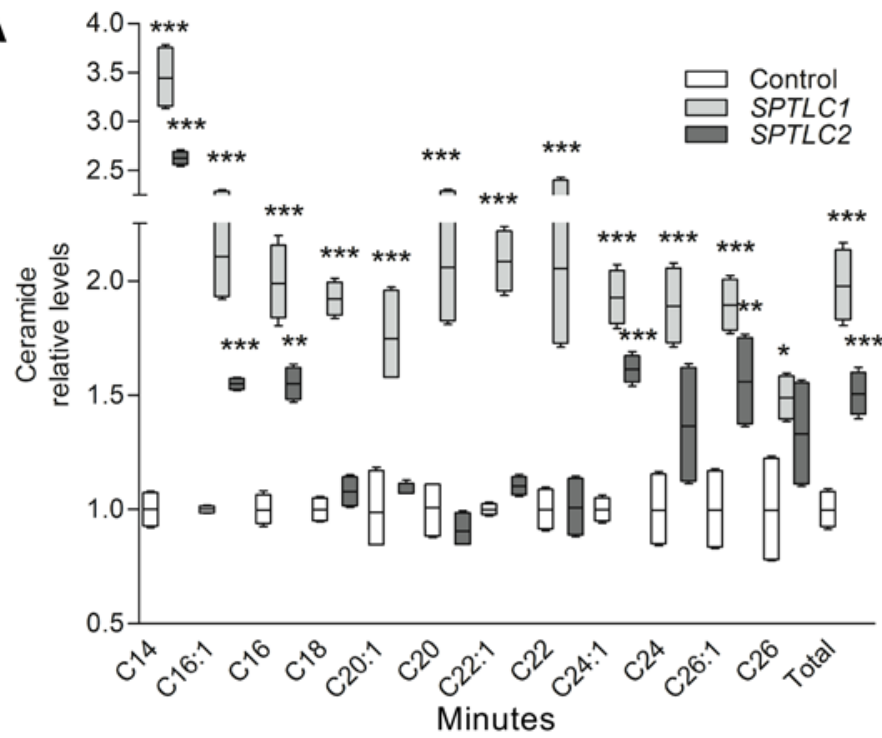

C



E
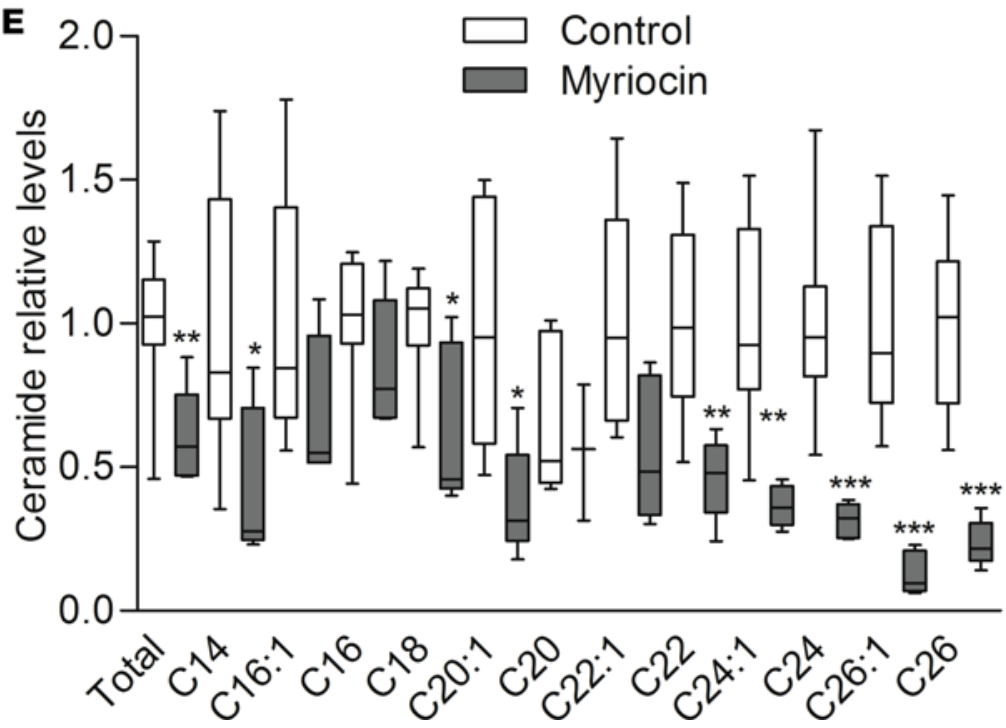

B Control

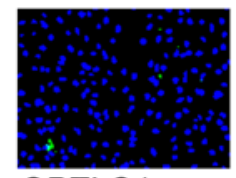

SPTLC1

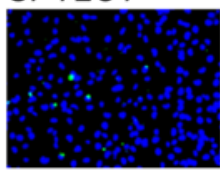

SPTLC2
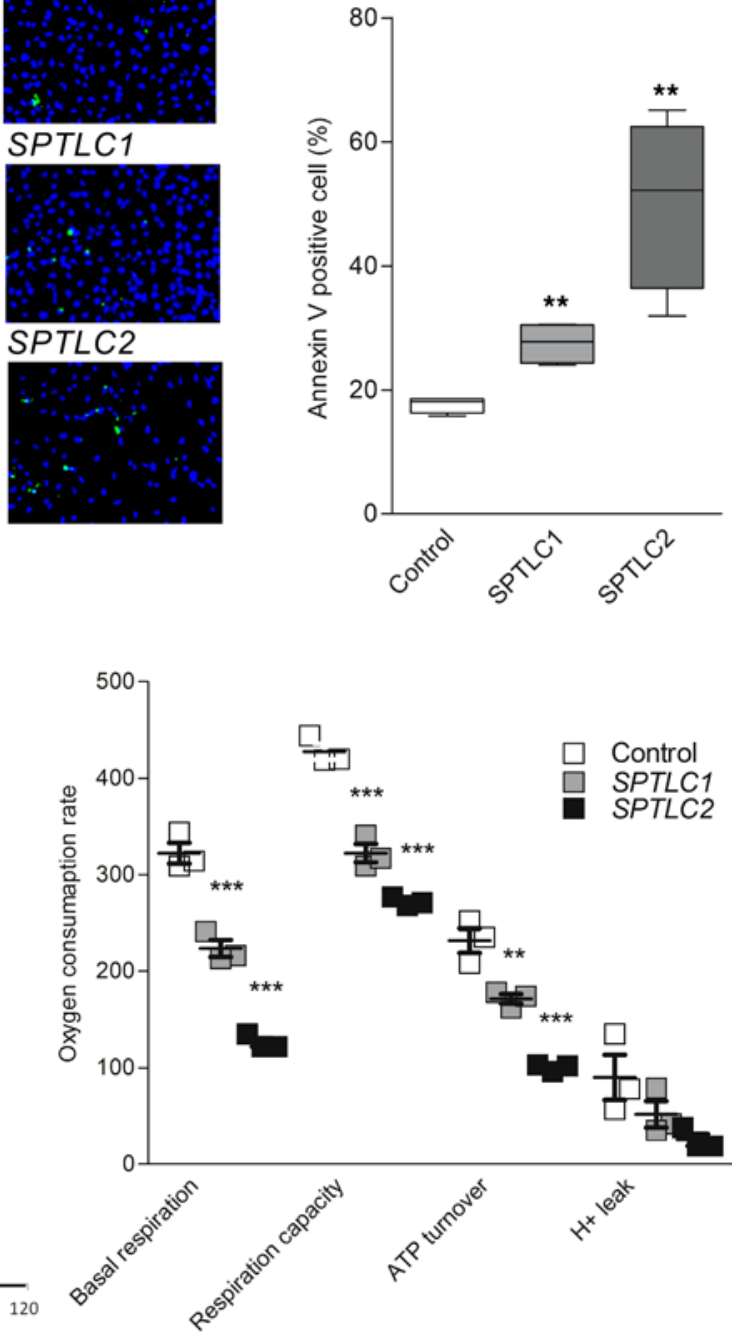

Figure 4. Effects of SPTLC1 and SPTLC2 overexpression on cellular ceramide levels, viability, and metabolism. (A) Increased levels of total and individual ceramide species in response to SPTLC1 and SPTLC2 overexpression in AC16 cells $(n=4)$. (B) Increased cellular apoptosis in response to SPTLC1 and -2 overexpression compared with controls. Percentage of apoptotic cells quantified by annexin-V staining $(n=4,20 \times)$. (C) Analysis of cellular oxidative metabolism by Seahorse analysis. Oxygen consumption rates were measured continuously throughout the experimental period starting at baseline and after addition of oligomycin (Oligo, $1 \mu \mathrm{M}$ ) and carbonylcyanide p-trifluoromethoxyphenylhydrazone (FFCP1, $1 \mu \mathrm{M}$ ). Rot, rotenone; anti, antimycin. (D) Averaged data of basal respiration, ATP turnover, $\mathrm{H}^{+}$leak, and respiratory capacity in AC16 cells in response to SPTLC1 and SPTLC2 overexpression and controls $(n=3)$. (E) Cellular ceramide levels in AC16 cells in response to myriocin treatment ( $n$ $=5-8$ per group). Two-tailed Student's $t$ test was used for 2 group comparisons, and one-way ANOVA was used for 3 group comparisons $\left({ }^{*} P<0.05\right.$, ${ }^{* *} P<0.01,{ }^{* * *} P<$ 0.001 versus control). 

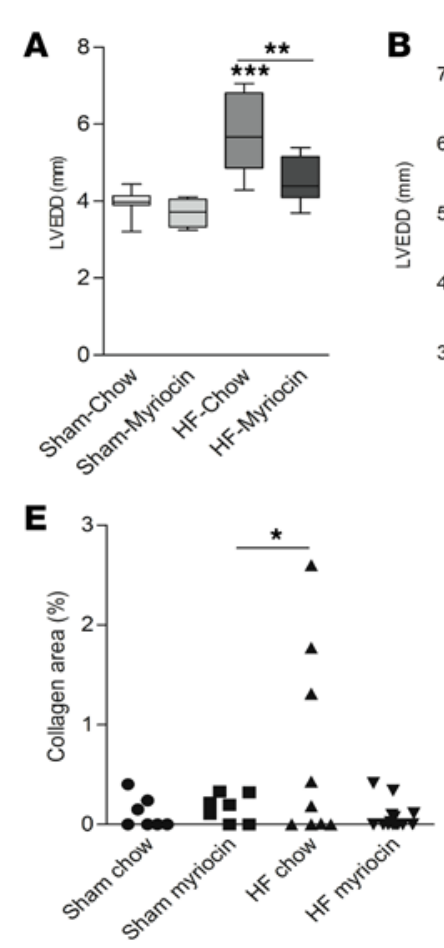

\section{H}

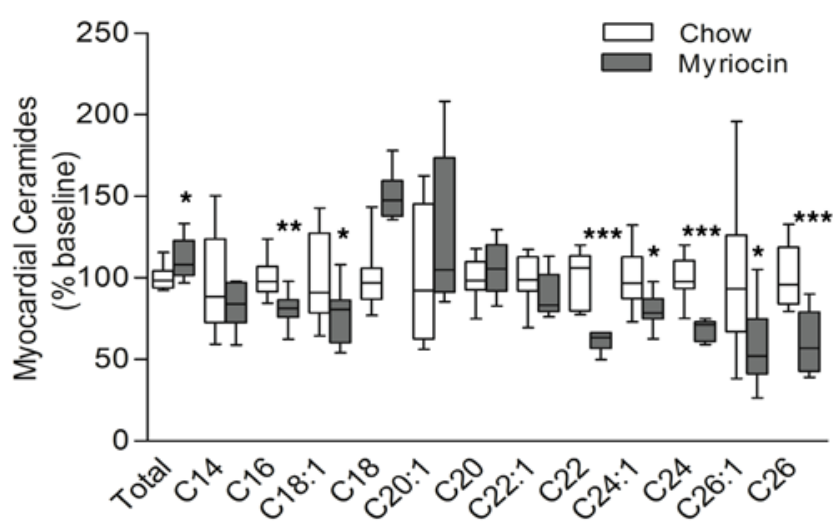

$\mathbf{J}$

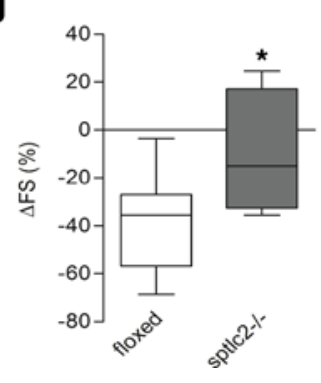

$\mathbf{K}$

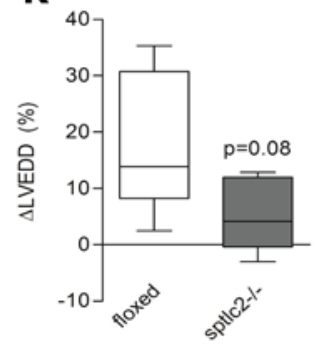

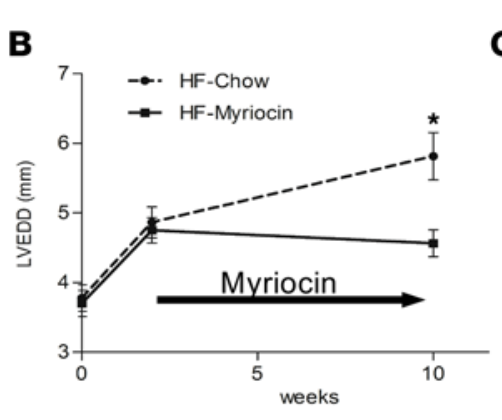

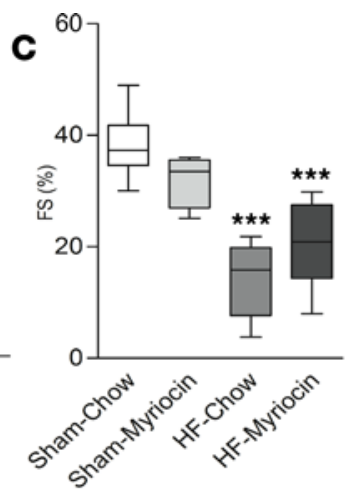

$\mathbf{F}$

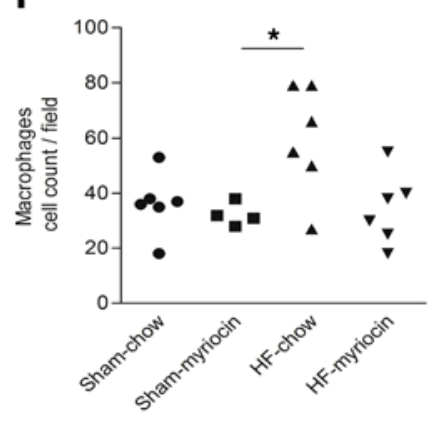

D

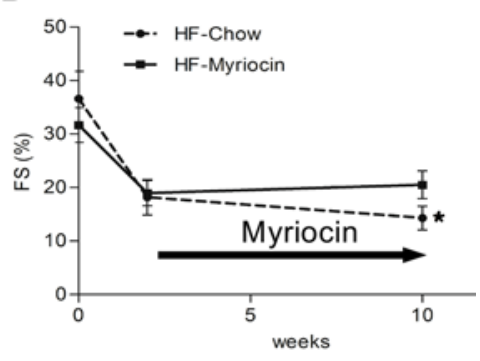

G

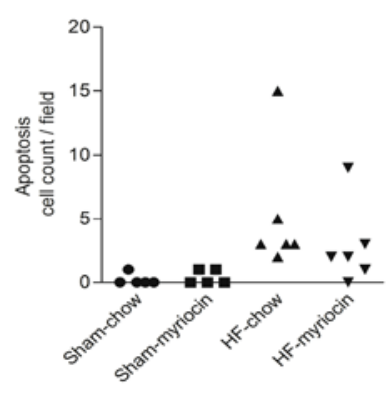

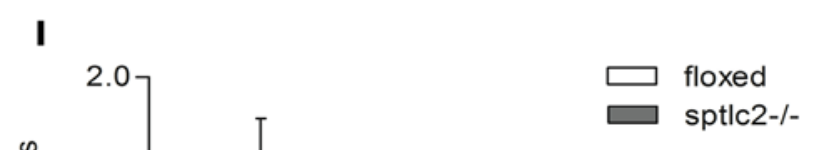

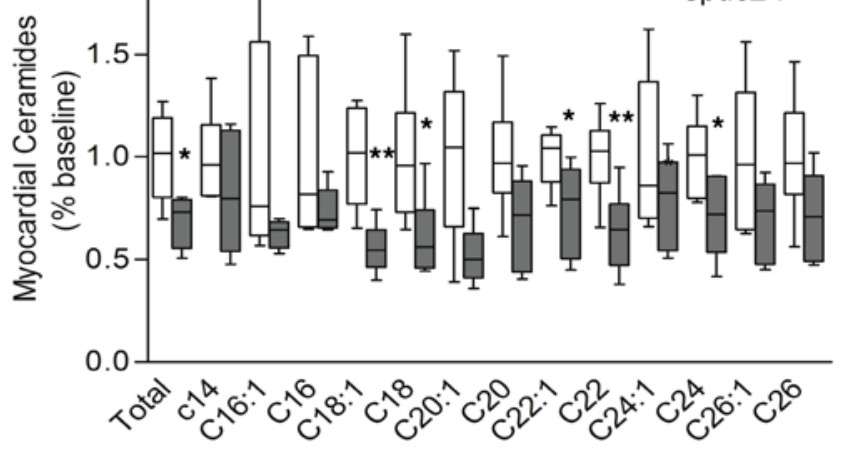


Figure 5. Effects of myriocin and Sptlc2 gene deletion on left ventricular remodeling following MI and of inhibition of ceramide synthesis in ischemic cardiomyopathy by myriocin on cardiac lipid composition. (A) Comparison of LVEDD in sham and MI on myriocin and placebo ${ }^{* *} P<0.01$, ${ }^{* * *} P<0.001$, compare to sham, $n=4-9$ ). (B) Dynamics in LVEDD following MI in animals treated with myriocin and placebo (change in LVEDD: ${ }^{*} P<0.05$ versus 2 weeks placebo) and change in LVEDD in MI placebo versus MI myriocin compared with 2 weeks after MI $(n=4-9)$. (C) Comparison of FS in sham and MI on myriocin and placebo $(n=4-9)$. (D) Dynamics in FS following MI in animals treated with myriocin and placebo at 10 weeks after MI compared with 2 weeks after MI (change in FS: ${ }^{*} P<0.05$ versus 2 weeks placebo, $n=4-9$ ). (E) Quantitative analysis of myocardial collagen staining using Masson's trichrome stain $\left(100 \times,{ }^{*} P<0.05\right.$ versus sham myriocin, $\left.n=7-14\right)$. (F) Macrophage staining using Mac3 antibody (100x, ${ }^{*} P<0.05$ versus sham myriocin, $\left.n=4-6\right)$. (C) Apoptosis staining using the TUNEL protocol (400x, $n=5-6)$. (H) Lipidomic analysis of total and individual ceramides in the noninfarcted myocardium 12 weeks after $\mathrm{MI}$ in animals treated with myriocin compared with placebo $\left({ }^{*} P<0.05,{ }^{*} P<0.01,{ }^{* *} P<0.001\right.$ versus chow, $\left.n=9\right)$. (I) Lipidomic analysis of total and individual ceramides in the Splt/c2-KO mice compared with floxed mice ( ${ }^{*} P<0.05,{ }^{* *} P<0.01$ versus floxed, $n=6$ ). (J) Preservation of FS following MI in Spt/c2-KO compared with control mice at 2 weeks after $\mathrm{MI}$ to 10 weeks after MI (change in FS: ${ }^{*} P<0.05$ ). (K) Reduced LV dilation following MI in Spt/c2KO compared with control mice from 2 weeks after MI to 10 weeks after MI (change in LVEDD: $P=0.08, n=5-8$ ). Two-tailed Student's $t$ test was used for 2 group comparisons, and one-way ANOVA was used for 3 group comparisons. LVEDD, left ventricular end diastolic diameter; FS, fraction shortening; MI, myocardial infarction.

protective effect in hypoxia (36). Alternatively, very long-chain ceramides have recently been associated with protection from liver injury and insulin resistance (37). Dynamics in the regulation of ceramides in hypoxia and under gemcitabine/doxorubicin treatment suggest that cellular pathways linked to the expression profiles of specific CERSs modulate certain ceramide species with partially opposing biologic effects (38). Indeed, gene deletion and overexpression models for various CERSs have shown differences in tissue-specific ceramide composition and content patterns (39).

A notable finding of our current study is the decrease in protein levels of distinct CERS2 with concomitant induction of SPTLC1 and SPTLC2 in the animal model of ischemic left ventricular dysfunction and in myocardium obtained from patients with advanced HF. Further, functional inhibition of the SPT enzyme complex increased expression levels of CERS1 and -5. This was accompanied by increased cardiac levels of C18 ceramide, a known product of CERS1 (30), but decreased levels of the very long-chain ceramides $\mathrm{C} 22$, C24, and C26. Of note, mechanical unloading through VAD implantation in human advanced HF resulted in identical changes on cardiac protein levels of SPTLCs and CERS, as well as ceramide levels. These findings suggest a pathway-specific variability of ceramide synthesis dynamics in relation to the underlying pathology and a distinct cellular response pattern.

In conclusion, total and very long-chain ceramides accumulate during the chronic remodeling process in the failing myocardium, and inhibition of de novo ceramide synthesis corrects abnormal ceramide content and composition. These findings provide a new understanding of systemic and myocardial lipid metabolism associated with chronic left ventricular dysfunction. Along with the well-established changes in cardiac oxidation of fatty acids, our data suggest that nonoxidized fatty acids, the substrate for the SPT de novo synthesis reaction, appear to be diverted into ceramide and perhaps also sphingomyelin synthesis. This might reflect a fundamental process to allow the heart to maintain essential lipid species in the setting of reduced uptake of circulating lipoprotein-derived sphingolipids. Alternatively, it might provide an escape loop to prevent accumulation of non-ATP-generating fatty acids. Whether pharmacologic interventions altering changes in ceramide synthesis pathways will prove to be beneficial in more human-like HF conditions will need to be tested.

\section{Methods}

Patient cohort. Sixty-five patients with advanced HF were recruited at Columbia University Medical Center. Of those, 31 were recruited from the outpatient clinics with stable HF, and 34 patients underwent implantation of a continuous-flow VAD. Twenty-seven patients were followed after VAD placement. Paired samples (pre- versus post-VAD placement) were available in 15 subjects; of these 15 patients pre- and post-VAD, $73 \%$ (11 of 15 patients) were patients with ischemic cardiomyopathy. Clinical, echocardiographic, and laboratory characteristics were collected from all patients at baseline and during followup.

Myocardial specimens were collected from a subset of patients $(n=15)$, and blood samples were obtained from all patients $(n=65)$ at the time of VAD implantation for end-stage HF as a bridge-to-transplantation and at the time of VAD explantation during cardiac transplantation. Blood was collected from stable subjects $(n=31)$ at the outpatient visit. Control blood samples were obtained from patients without cardiovascular disease $(n=22)$ recruited at Columbia University Medical Center. Control myocardial samples $(n=7)$ were obtained from deidentified specimens collected from nonfailing hearts that were determined to be unusable for cardiac transplantation due to acute recipient issues or donor coronary artery disease but were without evidence of previous infarction. 
Animal study. Ligation of the left descending coronary artery or sham surgery was performed in 69 C57B/L6 mice (Jackson Laboratory, age 10-12 weeks) and sptlc2-KO mice. Animals were anesthetized with a combination of ketamine (Sigma-Aldrich, $80 \mathrm{mg} / \mathrm{kg}$ ) and xylazine (Sigma-Aldrich, $10 \mathrm{mg} / \mathrm{kg}$ ) injected i.p. Pressure-controlled ventilation was initiated after intubation at $15 \mathrm{~cm} \mathrm{H}_{2} \mathrm{O}$. After chest opening, a 9-0 Prolene suture (tapered needle) was placed around the left anterior descending coronary artery 2 $\mathrm{mm}$ below the left atrium and ligated. Similar procedures were followed for sham surgery without ligation of the coronary artery. The chest was closed by suturing adjacent ribs and the skin.

WT mice following MI $(n=42)$ or sham surgery $(n=27)$ were randomized at week 2 to myriocin $(0.3$ $\mathrm{mg} / \mathrm{kg}$ ) or placebo (Chow Research Diets) treatment for a total of 8 weeks (sham placebo, $n=20$; sham myriocin, $n=7$; HF chow, $n=24$; and HF myriocin, $n=18$ ) to study the chronic effects of ceramide inhibition following $\mathrm{MI}$ and sham surgery. In an independent second study group, mice were pretreated with myriocin or placebo for 4 weeks before MI or sham surgery was performed. Animals were followed for 2 weeks before sacrifice to study the early effects following MI. Animals were housed in a temperature-controlled $\left(20^{\circ} \mathrm{C}-22^{\circ} \mathrm{C}\right)$ environment with a 12 -hour light/dark cycle. Blood was collected before sacrifice, centrifuged, and serum stored at $-80^{\circ} \mathrm{C}$. Noninfarcted tissue of the left ventricle was harvested at sacrifice. One part of the heart was placed in liquid nitrogen for lipid analysis, protein, and mRNA extraction; another part of heart was put into the $10 \%$ formalin in $4^{\circ} \mathrm{C}$ for 24 hours and then mounted on paraffin for histological analysis. Tissues were stored at $-80^{\circ} \mathrm{C}$.

Cardiomyocyte-specific Sptlc2 gene deletion. Cardiomyocyte-specific Sptlc2-deficient mice were previously created. Cardiomyocyte-specific Sptlc2-KO (Sptlc2-KO) mice were generated by using floxed Sptlc2 mice on a C57B1/6J background and crossed with $\alpha$-myosin heavy chain-Cre ( $\alpha \mathrm{MHC}-\mathrm{Cre})$ mice. Mice genotype was confirmed by PCR of the tissue extracts of tail.

Echocardiography. Cardiac function was assessed by echocardiography at baseline, 2 weeks after MI and before sacrifice to describe early and late remodeling. Mice were anesthetized with inhalational isoflurane anesthesia. Anesthesia was induced by $1.5 \%-2 \%$ isoflurane and was reduced to $0.5 \%-1 \%$ once the mouse was asleep. The chest skin of the mouse was shaved by using hair remover, and the heart was imaged by using a 30-MHz high frequency ultrasound transducer (Visualsonics Vevo770).

Cell culture. An established human cardiomyocyte-fibroblast fusion cell line (AC 16) was used for the study of cardiac signaling in vitro (40). All experiments were performed at least 3 times with positive and negative controls as described below. Hypoxia was induced in a hypoxic chamber using $2 \% \mathrm{O}_{2}$ and $5 \% \mathrm{CO}_{2}$ with $\mathrm{N}_{2}$ background for 3 hours. Experiments were performed for 3 hours in the presence or absence of myriocin $(1 \mathrm{uM})$. Apoptosis was detected using annexin-V staining (Invitrogen).

SPTLC1 and SPTLC2 overexpression plasmids. The full length of human SPTLC1 and SPTLC2 genes were constructed into the p3XFLAG-CMV-7 expression vector. Overexpression plasmids or p3XFLAG-CMV7-BAP (bacterial alkaline phosphatase, Sigma-Aldrich) control plasmid were transfected into cells using Lipofectamine 2000 (Invitrogen) as transfection reagent following the manufacturer's instructions.

Measurement of cellular oxygen consumption rates. The oxygen consumption rate (OCR) was determined using a Seahorse Bioscience XF24 Extracellular Flux analyzer at $37^{\circ} \mathrm{C}$. AC16 cells were plated on XF24 microplates (Seahorse Bioscience) at $5.0 \times 104$ cells/well in DMEM media supplemented with $10 \%$ FBS and kept at $37^{\circ} \mathrm{C}$ in a $5 \% \mathrm{CO}_{2}$ humidified atmosphere 24 hours before SPTLC1 and SPTLC2 overexpression. Intact cellular respiration was assayed at posttransfection 24 or 48 hours under basal conditions (10 mM D-glucose, $10 \mathrm{mM}$ pyruvate, $0 \%$ serum) and after the administration of various drugs as following: mitochondrial inhibitor oligomycin (oligo; $1 \mu \mathrm{M}$ ), mitochondrial uncoupler carbonylcyanide p-trifluoromethoxyphenylhydrazone (FCCP; $1 \mu \mathrm{M})$, respiratory chain inhibitor antimycin A (AA; $1 \mu \mathrm{M})$, and rotenone (ROT; $1 \mu \mathrm{M})$. The XF24 microplate was loaded into the Seahorse XF24 analyzer following the manufacturer's instructions.

Respiratory parameters were quantified by subtracting respiration rates at times before and after addition of electron transport chain inhibitors according to Seahorse Biosciences: basal respiration, baseline respiration minus AA-dependent respiration; ATP turnover, baseline respiration minus oligo-dependent respiration; $\mathrm{H}^{+}$leak, oligo-dependent respiration minus AA-dependent respiration; and respiratory capacity, FCCP-dependent respiration minus AA-dependent respiration. Values were calculated for each individual well and averaged for each condition.

Serum analysis. Serum lipid and glucose levels were measured using conventional methods (serum glucose tested by using OneTouch scanners, Life Scan Inc.). Serum FFAs were measured using HR Series NEFA-HR kit (2). Serum TG and TC were determined using the Infinity Triglycerides and Cholesterol kit (Thermo Fisher 
Scientific). Sample and standard was added into the working solution and incubated for 10 minutes and read.

LC/MS. All solvents for sample extraction and LC/MS were LC/MS grade (or LC grade when LC/ MS grade was not available) and were purchased from Thermo Fisher Scientific. Ceramide standards were purchased from Avanti Polar Lipid Inc.

Samples were extracted using chloroform/methanol extraction. Briefly, $3 \mathrm{ml}$ of the chloroform/methanol (2:1, v/v) containing $20 \mu 1$ of a $2 \mu \mathrm{M}$ internal standard mixture (Avanti LM-6002, containing C12 and C25 ceramides) were added to $100 \mu$ laqueous of tissue or cell homogenate in a clean glass tube. The mixture was vortexed well. Water $(0.5 \mathrm{ml})$ was added to the mixture to allow for phase separation. The mixture was vortexed again and centrifuged at 3,000 $\mathrm{g}$ for 10 minutes. The lower organic phase was transferred to a second clean glass tube using a Pasteur pipette. Chloroform $(2 \mathrm{ml})$ was added to the residual aqueous phase, followed by vortex mixing and centrifugation again at 3,000 $\mathrm{g}$ for 10 minutes, to extract the remaining lipids. The lower organic phases were pooled and evaporated under nitrogen. The extracted lipids were reconstituted in $30 \mu 1$ of methanol and transferred to LC/MS autosampler vials (Waters, P/N 600000670CV) for injection.

All experiments were carried out on a Waters Xevo TQ MS ACQUITY UPLC system. The system was controlled by Mass Lynx Software version 4.1. The sample was maintained at $4^{\circ} \mathrm{C}$ in the autosampler, and $5 \mu \mathrm{l}$ was loaded onto a Waters ACQUITY UPLC BEH Phenyl column $(3 \mathrm{~mm}$ inner diameter $\times 100$ $\mathrm{mm}$ with $1.7 \mu \mathrm{m}$ particles), preceded by a $2.1 \times 5 \mathrm{~mm}$ guard column containing the same packing. The column was maintained at $40^{\circ} \mathrm{C}$ throughout analysis. The UPLC flow rate was continuously $300 \mu 1 / \mathrm{min}$ in a binary gradient mode with the following mobile phase: initial flow conditions were $20 \%$ solvent $\mathrm{A}\left(\mathrm{H}_{2} \mathrm{O}\right.$, containing $0.2 \%$ formic acid and $1 \mathrm{mM}$ ammonium formate) and $80 \%$ solvent $\mathrm{B}$ (methanol, containing $0.2 \%$ formic acid and $10 \mathrm{mM}$ ammonium formate; ref. 41 ).

Western blot analysis. Total protein was extracted from cardiac samples with standard protein lysis buffer containing protease inhibitor cocktail (Roche Diagnostic). Protein content was determined using a protein assay kit (Thermo Fisher Scientific). The same amounts of protein were loaded to $4 \%-20 \%$ ready gels (Invitrogen) for electrophoresis, followed by transfer onto Immun-Blot Nitrocellulose membranes (Bio-Rad). After blocking, the membranes were incubated overnight at $4^{\circ} \mathrm{C}$ with the following primary antibodies: SPTLC1 (A303407A, Bethyl Laboratories Inc.), SPTLC2 (PA5-21142, Thermo Fisher Scientific), SPTLC3 (sc-86226, Santa Cruz Biotechnolgy Inc.), CERS5 (ab73289, Abcam), CERS2 (A70389, Sigma-Aldrich), ASM (sc-11352, Santa Cruz Biotechnolgy Inc.), CERS1 (QC18809, Sigma-Aldrich), and GAPDH (3683, Cell Signaling Technology). After washing and incubation with secondary antibody (sc-2030, goat anti-rabbit IgG-HRP from Santa Cruz Biotechnology Inc.) for 1 hour at room temperature, specific bands were visualized using super-signal west Pico chemiluminescense substrate reagent (Thermo Fisher Scientific) and developed by using Kodak BioMax MS films. The blot was scanned and analyzed by using the Quantity One image program.

Real-time PCR. RNA was extracted from mouse tissue samples using Trizol reagent by using standard protocols. Typically, 5-10 $\mu \mathrm{g}$ of total RNA was obtained from cardiac samples. Samples were stored at $-80^{\circ} \mathrm{C}$. Multiplexed amplification reactions were performed using $\beta$-actin mRNA as an endogenous control, using the Super Script III First-Stand Synthesis SuperMix kit (Invitrogen). Specific primers were designed for Sptlc1, Sptlc2, Asm, Cers1, Cers2, Cers5, Bcl2, Bcl-XL, Chop, and Bax, which were purchased directly from Invitrogen (see Supplemental Table 1; supplemental material available online with this article; https://doi.org/10.1172/jci.insight.82922DS1). RNA samples were analyzed in triplicate, and the mean value was calculated. For gene expression detection, SYBR Green detection (Agilent) was used. Values were log-transformed before statistical analysis.

Histological analysis. Paraffin-embedded tissues were cut $(5 \mu \mathrm{m})$ and mounted onto microslides. Following deparaffinization, antigen retrieval was performed by heating in Citra plus (Biogenex) for 20 minutes. We used $3 \%$ hydrogen peroxide for 10 minutes to block endogenous peroxidase. Primary and secondary antibodies were incubated using standard protocols. Macrophages were detected using primary antibody Mac3 and secondary antibody goat anti-rat (BD Biosciences, catalog 550767). Specific immunoreactivity was detected using avidin-biotin conjugation and DAB for staining followed by counterstaining with hematoxylin. Myocardial collagen changes were detected using Masson's trichrome staining. Apoptotic nuclei were detected using the TUNEL reaction (TACS2 TdT DAB in-situ Apoptosis Detection Kit).

Statistics. Results are expressed as mean \pm SEM. Probability values of $P<0.05$ were considered significant. Comparison among all groups was calculated and statistically compared using 2-tailed Student's $t$ test or ANOVA with significance defined as $P<0.05$. Statistical analysis was performed using GraphPad Prism 5 (GraphPad Software Inc.). 
Study approval. All animal protocols were approved by the Columbia University Institutional Animal Care and Use Committee. All human studies were approved by the Columbia University IRB, and written informed consent was obtained from participants prior to inclusion in the study.

\section{Author contributions}

RJ, HA, XL, and HJ performed experiments; RJ, XL, HJ, XZ, KD, PJK, DLB, EC, LYD, SH, I.J. Goldberg, HT, and YN interpreted results of experiments; RJ, PK, and DLB prepared figures; RJ drafted the manuscript; PCS and I.J. Goldberg edited and revised the manuscript; PCS helped with conception and design of research; I.J. George helped design the experiments, collect samples from patients and controls, analyze the data, and revise the manuscript; all authors approved the final version of manuscript.

\section{Acknowledgments}

This study was supported by grants from the NIH to PCS (K23 HL095742-01, P30 HL101272-01 and RO1 HL114813) and I.J. Goldberg (RO1 HL73029). KD was supported by a "Pathway to Independence" Award 1K99HL112853 from the NHLBI. DLB was supported by a T32 training grant from the NIH. Sptlc2 gene deletion mice were provided by Xian-cheng Jiang, Downstate Medical Center, Brooklyn, New York, USA.

Address correspondence to: P. Christian Schulze, Department of Internal Medicine I, Division of Cardiology, Angiology, Pneumology and Intensive Medical Care, University Hospital Jena, Friedrich-Schiller-University Jena, Am Klinikum 1, 07747 Jena, Germany. Phone: 49.3641.9324101; E-mail: christian.schulze@ med.uni-jena.de.

1. Writing Committee Members, et al. ACCF/AHA/ACP/HFSA/ISHLT 2010 clinical competence statement on management of patients with advanced heart failure and cardiac transplant: a report of the ACCF/AHA/ACP Task Force on Clinical Competence and Training. Circulation. 2010;122(6):644-672.

2. Goldberg IJ, Trent CM, Schulze PC. Lipid metabolism and toxicity in the heart. Cell Metab. 2012;15(6):805-812.

3. Schulze PC, Drosatos K, Goldberg IJ. Lipid Use and Misuse by the Heart. Circ Res. 2016;118(11):1736-1751.

4. Chatham JC, Young ME. Regulation of myocardial metabolism by the cardiomyocyte circadian clock. J Mol Cell Cardiol. 2013;55:139-146.

5. Lopaschuk GD, Ussher JR, Folmes CD, Jaswal JS, Stanley WC. Myocardial fatty acid metabolism in health and disease. Physiol Rev. 2010;90(1):207-258.

6. Razeghi P, Young ME, Alcorn JL, Moravec CS, Frazier OH, Taegtmeyer H. Metabolic gene expression in fetal and failing human heart. Circulation. 2001;104(24):2923-2931.

7. Taegtmeyer H, Sen S, Vela D. Return to the fetal gene program: a suggested metabolic link to gene expression in the heart. Ann N Y Acad Sci. 2010;1188:191-198.

8. Chokshi A, et al. Ventricular assist device implantation corrects myocardial lipotoxicity, reverses insulin resistance, and normalizes cardiac metabolism in patients with advanced heart failure. Circulation. 2012;125(23):2844-2853.

9. Sharma S, et al. Intramyocardial lipid accumulation in the failing human heart resembles the lipotoxic rat heart. FASEB J. 2004;18(14):1692-1700.

10. Drosatos K, et al. Cardiomyocyte lipids impair $\beta$-adrenergic receptor function via PKC activation. Am J Physiol Endocrinol Metab. 2011;300(3):E489-E499.

11. Tuunanen $\mathrm{H}$, et al. Free fatty acid depletion acutely decreases cardiac work and efficiency in cardiomyopathic heart failure. Circulation. 2006;114(20):2130-2137.

12. Tuunanen $\mathrm{H}$, et al. Decreased myocardial free fatty acid uptake in patients with idiopathic dilated cardiomyopathy: evidence of relationship with insulin resistance and left ventricular dysfunction. J Card Fail. 2006;12(8):644-652.

13. Zhou YT, et al. Lipotoxic heart disease in obese rats: implications for human obesity. Proc Natl Acad Sci USA. 2000;97(4):1784-1789.

14. Oral H, Dorn GW, Mann DL. Sphingosine mediates the immediate negative inotropic effects of tumor necrosis factor-alpha in the adult mammalian cardiac myocyte. J Biol Chem. 1997;272(8):4836-4842.

15. Hunter JJ, Chien KR. Signaling pathways for cardiac hypertrophy and failure. N Engl J Med. 1999;341(17):1276-1283.

16. Hla T, Dannenberg AJ. Sphingolipid signaling in metabolic disorders. Cell Metab. 2012;16(4):420-434.

17. Park TS, et al. Ceramide is a cardiotoxin in lipotoxic cardiomyopathy. J Lipid Res. 2008;49(10):2101-2112.

18. Bielawska AE, et al. Ceramide is involved in triggering of cardiomyocyte apoptosis induced by ischemia and reperfusion. Am $J$ Pathol. 1997;151(5):1257-1263.

19. Lee SY, et al. Cardiomyocyte specific deficiency of serine palmitoyltransferase subunit 2 reduces ceramide but leads to cardiac dysfunction. J Biol Chem. 2012;287(22):18429-18439.

20. Delpy E, et al. Doxorubicin induces slow ceramide accumulation and late apoptosis in cultured adult rat ventricular myocytes. Cardiovasc Res. 1999;43(2):398-407.

21. Pchejetski D, et al. Oxidative stress-dependent sphingosine kinase-1 inhibition mediates monoamine oxidase A-associated cardiac cell apoptosis. Circ Res. 2007;100(1):41-49.

22. Hernandez OM, Discher DJ, Bishopric NH, Webster KA. Rapid activation of neutral sphingomyelinase by hypoxia-reoxygen ation of cardiac myocytes. Circ Res. 2000;86(2):198-204. 
23. Bose R, Verheij M, Haimovitz-Friedman A, Scotto K, Fuks Z, Kolesnick R. Ceramide synthase mediates daunorubicin-induced apoptosis: an alternative mechanism for generating death signals. Cell. 1995;82(3):405-414

24. Verheij M, et al. Requirement for ceramide-initiated SAPK/JNK signalling in stress-induced apoptosis. Nature. 1996;380(6569):75-79.

25. E1 Btaouri H, Morjani H, Greffe Y, Charpentier E, Martiny L. Role of JNK/ATF-2 pathway in inhibition of thrombospondin-1 (TSP-1) expression and apoptosis mediated by doxorubicin and camptothecin in FTC-133 cells. Biochim Biophys Acta. 2011;1813(5):695-703

26. Thayyullathil F, et al. Glutathione selectively inhibits Doxorubicin induced phosphorylation of p53Ser ${ }^{15}$, caspase dependent ceramide production and apoptosis in human leukemic cells. Biochem Biophys Res Commun. 2011;411(1):1-6.

27. Kato TS, et al. Effects of continuous-flow versus pulsatile-flow left ventricular assist devices on myocardial unloading and remodeling. Circ Heart Fail. 2011;4(5):546-553.

28. Khan RS, et al. Adipose tissue inflammation and adiponectin resistance in patients with advanced heart failure: correction after ventricular assist device implantation. Circ Heart Fail. 2012;5(3):340-348

29. Yang JA, et al. Liver dysfunction as a predictor of outcomes in patients with advanced heart failure requiring ventricular assist device support: Use of the Model of End-stage Liver Disease (MELD) and MELD eXcluding INR (MELD-XI) scoring system. J Heart Lung Transplant. 2012;31(6):601-610.

30. Sentelle RD, et al. Ceramide targets autophagosomes to mitochondria and induces lethal mitophagy. Nat Chem Biol. 2012;8(10):831-838

31. Park TS, et al. Inhibition of sphingomyelin synthesis reduces atherogenesis in apolipoprotein E-knockout mice. Circulation. 2004;110(22):3465-3471.

32. Dekker MJ, et al. Inhibition of sphingolipid synthesis improves dyslipidemia in the diet-induced hamster model of insulin resistance: evidence for the role of sphingosine and sphinganine in hepatic VLDL-apoB100 overproduction. Atherosclerosis 2013;228(1):98-109.

33. Torre-Amione G, et al. Tumor necrosis factor-alpha and tumor necrosis factor receptors in the failing human heart. Circulation. 1996;93(4):704-711

34. Taha TA, Mullen TD, Obeid LM. A house divided: ceramide, sphingosine, and sphingosine-1-phosphate in programmed cell death. Biochim Biophys Acta. 2006;1758(12):2027-2036.

35. Noureddine L, et al. Modulation of total ceramide and constituent ceramide species in the acutely and chronically hypoxic mouse heart at different ages. Prostaglandins Other Lipid Mediat. 2008;86(1-4):49-55.

36. Menuz V, et al. Protection of C. elegans from anoxia by HYL-2 ceramide synthase. Science. 2009;324(5925):381-384.

37. Turpin SM, et al. Obesity-induced CerS6-dependent C16:0 ceramide production promotes weight gain and glucose intolerance. Cell Metab. 2014;20(4):678-686.

38. Senkal CE, et al. Role of human longevity assurance gene 1 and C18-ceramide in chemotherapy-induced cell death in human head and neck squamous cell carcinomas. Mol Cancer Ther. 2007;6(2):712-722.

39. Pewzner-Jung Y, et al. A critical role for ceramide synthase 2 in liver homeostasis: I. alterations in lipid metabolic pathways. $J$ Biol Chem. 2010;285(14):10902-10910.

40. Davidson MM, et al. Novel cell lines derived from adult human ventricular cardiomyocytes. J Mol Cell Cardiol. 2005;39(1):133-147.

41. Clugston RD, et al. Altered hepatic lipid metabolism in C57BL/6 mice fed alcohol: a targeted lipidomic and gene expression study. J Lipid Res. 2011;52(11):2021-2031. 